\title{
The two-layer skirted island
}

\author{
by Joseph Pedlosky ${ }^{1,2}$, Roberto Iacono ${ }^{3}$, Ernesto Napolitano ${ }^{3}$ and Michael A. Spall ${ }^{1}$
}

\begin{abstract}
The flow around a planetary scale island in a baroclinic ocean is examined when the island possesses a topographic skirt representing a steep continental slope and the ocean is modeled as a two-layer system in order to examine the role of stratification in the circulation. The study extends an earlier barotropic model of similar geometry and forcing to focus on the degree to which the topography, limited here to the lower of the two layers, affects the circulation and to what degree the circulation is shielded by stratification from the topographic effects noted in the simpler barotropic model.

As in the barotropic model, the topography is steep enough to produce closed, ambient potential vorticity contours over the topography in the lower layer providing free "highways" for the deep flow in the presence of small forcing by the wind-driven upper layer flow. The flow is very weak outside the region of closed contours but can become of the same order, if somewhat smaller, as the upper layer flow on those contours in the presence of even weak coupling to the upper layer.

A series of models, analytical and numerical, are studied. Linear theory is applied to two configurations. The first consists of a long, meridionally oriented island with a topographic skirt in the lower layer. The lower layer flow is driven by a hypothesized frictional coupling between the two layers that depends on the circulation of the upper layer velocity on a circuit defined by the closed potential vorticity contours of the lower layer. The largest part of the driving flow is identical on both sides of the island and cancels in the contour integration. The major part of the residual forcing comes from relatively small but effective forcing on the semi-circular tips of the topographic skirt. A circular island with a topographic skirt is also examined in which the coupling to the upper layer is stronger all around the island. Even in this case there is a delicate balance of the forcing of the lower layer on each side of the island. In all cases the flow on closed potential vorticity contours in the lower layer is much weaker than in the barotropic model but much stronger than in the flat region of the lower layer.
\end{abstract}

A sequence of numerical calculations that both check and extend the analytic linear theory is presented demonstrating the subtlety of the force balances. Further nonlinear, eddy-containing experiments give a preview of the direction of future work.

\section{Introduction}

In a recent paper, Pedlosky et al. (2009), hereafter PINH, considered the flow around a planetary scale island when the island possesses a topographic skirt that girdles the island

1. Woods Hole Oceanographic Institution, Woods Hole, Massachusetts, 02543, USA.

2. Corresponding author. email:jpedlosky@whoi.edu

3. ENEA, C. R. Casaccia, Rome Italy. 
and crudely represents a continental slope. Of particular interest was the change in both the structure of the circulation near the island and the way in which Godfrey's (1989) "Island Rule" must be reinterpreted. The presence of isolines of ambient potential vorticity, $f / h$, where $f$ is the Coriolis parameter and $h$ is the depth of the single, constant density layer of the model, completely alters the form of the circulation. It eliminates the recirculation found in theories of flat bottom oceans (Pedlosky et al., 1997) and traps a relatively strong circulation around the potential vorticity (pv) contours that does not participate in the basinwide circulation and suggests that the Island Rule should instead be applied to the domain demarked by the outermost closed pv contour instead of the boundary of the island's surface expression. The strong circulation on the closed pv contours represents a steady resonance, that would exist in a frictionless fluid, of the free geostrophic mode on those contours. The resonance, forced by the wind stress in the model, is bounded ultimately by the weak dissipation in the system and so is able to reach large velocities.

It is naturally of interest to reconsider the problem to take into account the baroclinic character of the ocean circulation and to examine to what extent the ideas of the barotropic model of PINH remain valid when the topography is shielded from the direct forcing by the wind by an overlying layer of lower density. We take up this problem in a two-layer model to keep our models, both analytic and numerical, as simple as possible. In this paper the topography will be limited to the region of the lower, second layer although we shall discuss qualitatively some results for larger topography that penetrates into the upper layer. Some previous works using wind-driven, baroclinic ocean models have examined the island effect (e.g. Pedlosky, 2010; Spall, 2000), but the joint effect of topography and stratification renders the dynamics considerably more complex. Dewar (1998) considered the deep flow over regions of closed potential vorticty contours, and found that eddy fluxes could drive strong barotropic recirculations in which forcing is balanced by bottom drag.

Section 2 describes the basic model and the model equations, which are essentially a two-layer version of the dynamics in PINH. Section 3 describes the basic model geometries and the results of linear theory for the two geometries considered, i.e. the meridional, thin island and skirt and the circular island with its skirt that girdles the island. In both cases the topography introduces closed isolines of potential vorticity in the lower of the two layers. The forcing of the lower layer flow is shown to be much less efficient for the meridionally oriented island over most of its length. Nevertheless, effective forcing over a relatively small domain near the two meridional extremes of the topography dominate the forcing and are sufficient to drive a substantial flow along the pv contours in the lower layer. The circular island is studied as an example of geometry where the forcing is more effective over a larger portion of the skirt. Section 4 presents our numerical results and compares them with the results of our simple analytical theory and this serves to underline the delicate nature of those analytical results reflecting the subtle balances that obtain in the dynamics. Section 5 presents some preliminary results where layer coupling is due to spontaneously generated eddies. In section 6 we summarize and discuss our results. 


\section{The model}

Figure 1 shows the geometry of the first model we are using. The first panel shows a zonal cross section of the island and its topography. In the upper layer it is a very thin island oriented in the north-south direction for $y_{s} \leq y \leq y_{n}$. The height of the peak of the topography from the otherwise flat bottom is $h_{T}$, and it extends a distance $x_{T}$ to each side of the island. The local thickness of the second layer is $h_{2}$ while the layer thicknesses beyond the topography are, in the absence of motion, $H_{1}$ and $H_{2}$, both constant. In plan view its topography is shown in Figure $1 \mathrm{~b}$ while the third panel shows the isolines of ambient potential vorticity, i.e. in the absence of motion, for a skirt width of $250 \mathrm{~km}$, a height above the bottom of $500 \mathrm{~m}$ in a layer of thickness 1000 meters which is also the thickness of the upper layer. This relatively short island is $800 \mathrm{~km}$ in length. For these parameters there is significant departure of the $\mathrm{pv}$ isolines from the topography but we will generally consider cases in which the departure is locally rather small. Even in this case the pv gradient due to the beta effect is ten times smaller that the pv gradient due to the change in layer thickness induced by the topography. Note that the topography has been smoothed within semi-circular regions at the northern and southern tips of the island to avoid abrupt changes in depth.

The second geometry we will examine, shown in Figure 2, consists of a circular island with radius $r_{I}$ that in the lower layer is surrounded by an azimuthally symmetric, circular skirt that extends to radius $r_{T}$. As in the previous case, the skirted island is placed at the center of a basin of radius $r_{o}$.

The circulation is driven by a wind stress,

$$
\vec{\tau}=\rho \tau_{o} r / r_{o} \hat{i}_{\theta},
$$

where $\hat{i}_{\theta}$ is a unit vector in the azimuthal direction, and $r$ is the radial distance from the center of our circular ocean basin. The mean density, $\rho$, is included in the definition of the stress to simplify the formulae that follow so that $\tau_{o}$ has the dimensions of a velocity squared. Note that the curl of $\vec{\tau} / \rho$ is spatially constant, and equals $2 \tau_{o} / r_{o}$.

For our governing equations we take, for each layer,

$$
\begin{gathered}
\frac{\partial \vec{u}_{n}}{\partial t}+\left(\zeta_{n}+f\right) \vec{k} \times \vec{u}_{n}=-\nabla B_{n}+\frac{\vec{\tau}}{\rho h_{1}} \delta_{n 1}+\vec{D}_{n}\left(\vec{u}_{1}, \vec{u}_{2}\right), n=1,2 \\
\frac{\partial h_{n}}{\partial t}+\nabla \cdot\left(h_{n} \vec{u}_{n}\right)=0,
\end{gathered}
$$

where

$$
\begin{aligned}
& B_{n}=\frac{1}{2}\left|\vec{u}_{n}\right|^{2}+p_{n} / \rho, \quad \zeta_{n}=\vec{k} \cdot \nabla \times \vec{u}_{n} \\
& \vec{D}_{n}\left(\vec{u}_{1}, \vec{u}_{2}\right)=\left(\frac{\lambda_{i}}{h_{n}}\right)\left(\vec{u}_{1}-\vec{u}_{2}\right)(-1)^{n}-\left(\frac{\lambda}{h_{2}}\right) \vec{u}_{2} \delta_{n 2}+\frac{1}{h_{n}}\left(\nabla \cdot A h_{n} \nabla\right) \vec{u}_{n}
\end{aligned}
$$


a)
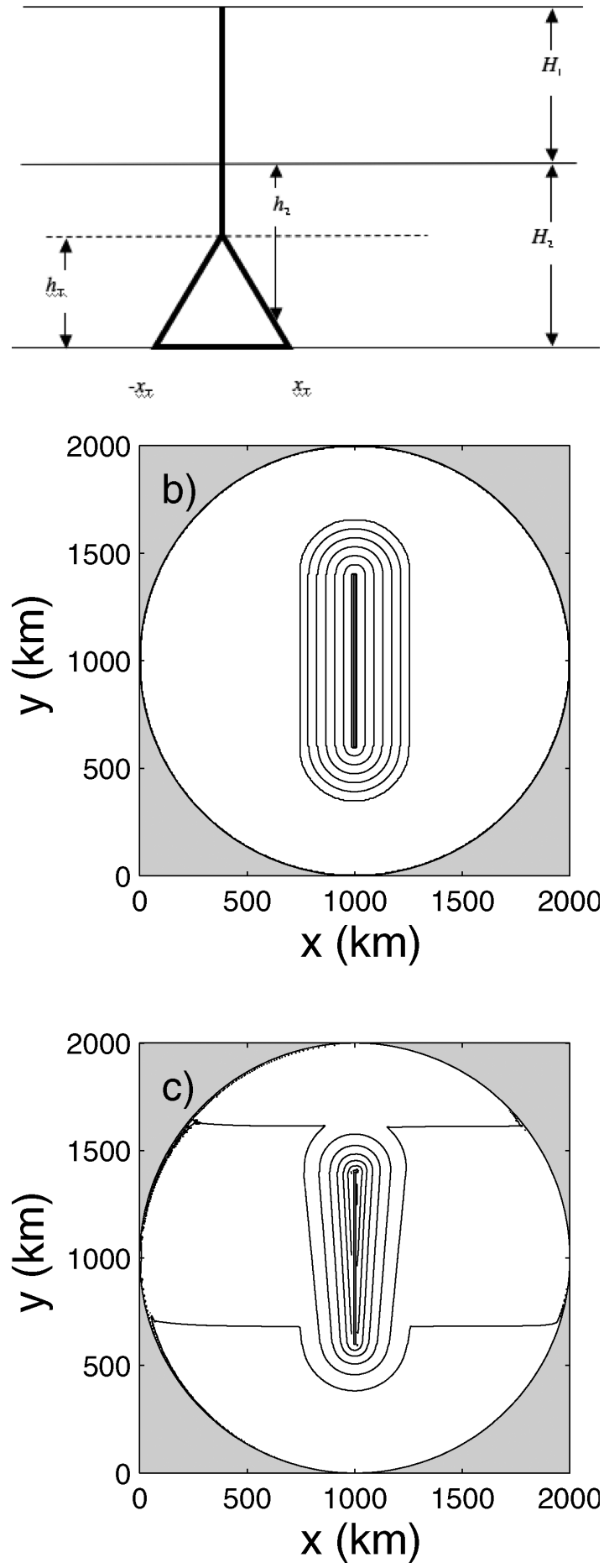

Figure 1. (a) The two-layer model showing the topography and layer thicknesses for the meridionally oriented island. (b) The depth contours in units of 100 meters. (c) The isolines of potential vorticity 


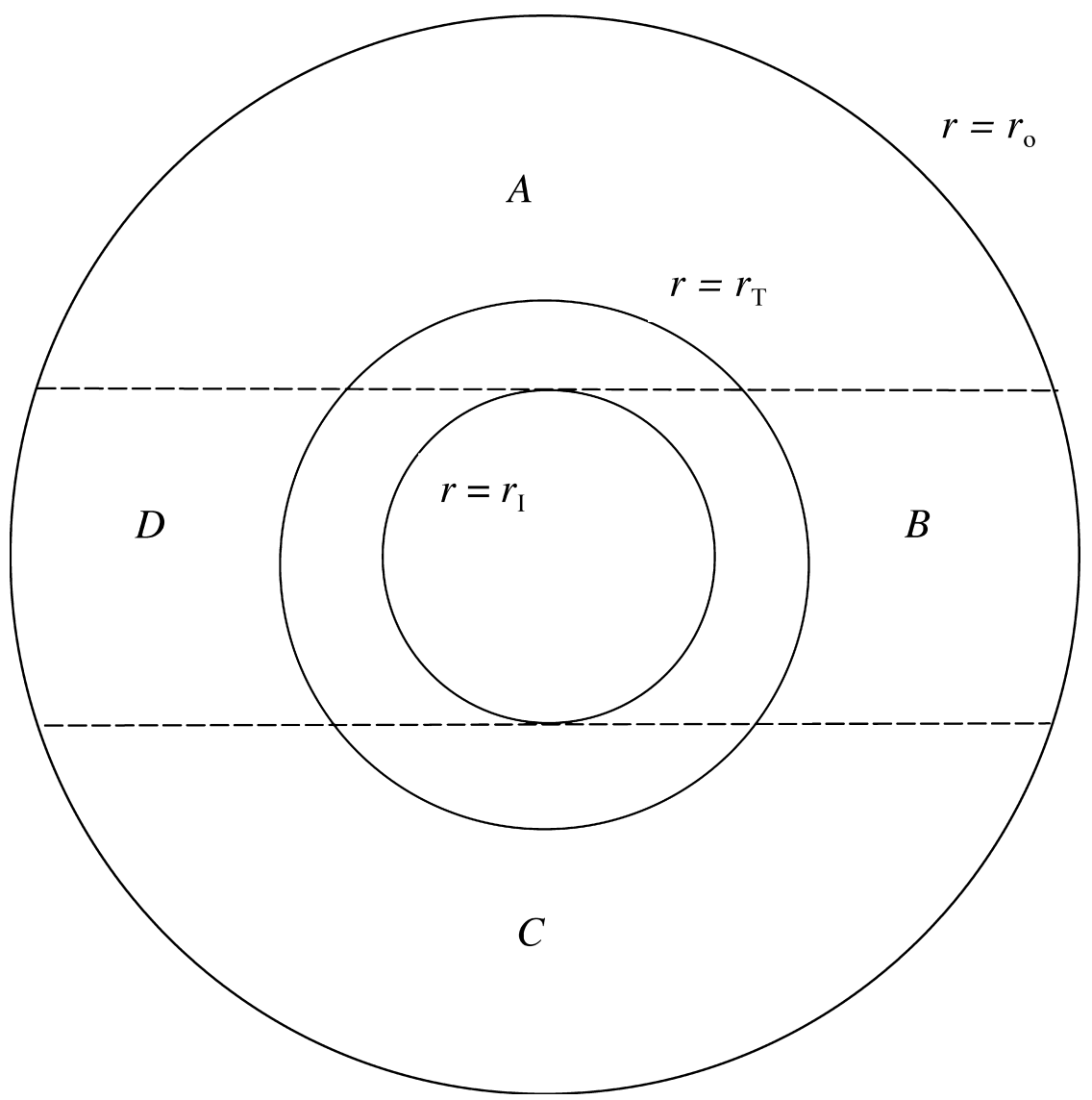

Figure 2. The four regions for the upper layer Sverdrup solution showing the island with inner radius $r_{I}$ in the upper layer and the skirt in the lower layer extending to $r_{T}$. For the meridional island the inner circle is replaced by a north-south line.

where $\delta_{n m}$ is the Kronecker delta, and $f=f_{0}+\beta y$ is the Coriolis parameter, which takes the value $f_{0}$ at $y=0$, the midlatitude of the basin.

The equations are nearly the same as used in PINH. These are, in (2.2a), the horizontal momentum equations for each layer. The stress appears as a body force in layer 1 ; that term is divided by the layer thickness since it is really a surface stress. The dissipation consists of the three terms given in (2.3b). The first is an interfacial friction term whose coefficient is $\lambda_{i}$. It exerts an equal and opposite stress on each layer proportional to the velocity difference. This vertical momentum flux is consistent with a parameterization of baroclinic instability as a horizontal diffusion of layer thickness (e.g., Gent et al., 1995). The second term is a bottom friction term proportional to $\lambda$ again divided by the second layer thickness. The remaining term is our representation of lateral momentum mixing. We have used this model 
of interfacial friction to couple the two layers instead of a cross isopycnal velocity so that in the steady state we can represent the horizontal transport by a streamfunction since our equation for mass conservation (2.2b) has no cross isopycnal velocity. Thus, for steady flows (2.2b) is identically satisfied by the representation in terms of the streamfunction $\psi_{n}$,

$$
\vec{u}_{n} h_{n}=\hat{k} \times \nabla \psi_{n}, n=1,2
$$

where $h_{n}$ are the actual thicknesses of the layers including the variations due to topography. In keeping with the linear approximation, we neglect variations in layer thickness due to sloping isopycnals associated with the vertical shear in horizontal velocity. This is valid for $\varepsilon\left(L / L_{d}\right)^{2} \ll 1$ here $\varepsilon=U / f L$, and $L$ is a characteristic width of the topography.

For steady motions the momentum equations can be usefully written as

$$
q_{n} \nabla \psi_{n}=\nabla B_{n}-\frac{\vec{\tau}}{h_{1}} \delta_{n 1}-\vec{D}_{n}\left(\vec{u}_{1}, \vec{u}_{2}\right)
$$

where $q_{n}=\left(\zeta_{n}+f\right) / h_{n}$ is the potential vorticity of layer $n$.

The vorticity equation for the lower layer combined with the mass conservation equation for the layer yields the potential vorticity equation for layer 2 which for a steady state is,

$$
\vec{u}_{2} \cdot \nabla q_{2}=\frac{1}{h_{2}} \vec{k} \cdot \nabla \times \vec{D}_{2}
$$

so that for weak enough dissipation, we would anticipate the potential vorticity for layer 2 to be locally conserved. Furthermore, if there is a closed contour of potential vorticity, $q_{2}$, in the lower layer an integral of (2.5) around that contour implies that

$$
\oint_{C q_{2}} \vec{D}_{2} \cdot \vec{t} d s=0 .
$$

This constraint holds for all nonlinearity but it will be most usefully employed in the linear theory of the next section where the contours of constant potential vorticity are the ambient isolines of $q_{2}=f / h_{2}$ and $h_{2}$ is known.

\section{Linear theory}

To help establish a conceptual picture of the dynamics in the two-layer model it is useful to consider the situation when the wind stress is weak enough to produce a slow, laminar circulation in the upper layer. We will also examine the case where the dissipation is very weak. In particular, we will avoid regions in which the lateral mixing is important, i.e. we will remain outside Munk layers, whose thickness we can estimate as $\delta_{M}=(A / \beta)^{1 / 3}$ and we consider the case where $\delta_{M} \ll x_{T}, \delta_{M} \ll r_{T}-r_{I}$. Over regions of closed potential vorticity contours the thickness generally would be of the order of $\left(A h_{2} / \lambda\right)^{1 / 2}$ via (2.7) with a balance between the second and third terms of (2.3). 
The interfacial friction coefficient $\lambda_{i}$ and the bottom friction coefficient $\lambda$ are also taken as small, i.e.

$$
\left(\lambda, \lambda_{i}\right) / \beta h_{2} \ll\left(x_{T}, r_{T}-r_{I}\right) .
$$

When the contours, $C_{q 2}$, of $f / h_{2}$ are closed, i.e. when the topographic contribution to the potential vorticity in layer two is large enough, the constraint (2.7) applies on those contours (see (3.5c) and (3.17) below). Further, if the coefficients of bottom and interfacial friction satisfy (3.1) the constraint (2.7) implies

$$
\lambda_{i} \oint_{C_{q 2}} \frac{\nabla \psi_{1} \cdot \vec{n}}{h_{1} h_{2}} d s=\left(\lambda+\lambda_{i}\right) \oint_{C_{q 2}} \frac{\nabla \psi_{2} \cdot \vec{n}}{h_{2}^{2}} d s
$$

on each such contour and the potential vorticity equation implies in the linear limit that those isolines are given by the isolines of $f / h_{2}$ which are known a priori. The potential vorticity equation in turn implies that

$$
\psi_{2}=\Psi_{2}\left(q_{2}\right)
$$

or from (3.2)

$$
\frac{d \Psi_{2}}{d q_{2}}=\frac{\lambda_{i}}{\lambda+\lambda_{i}} \frac{\mathscr{C}_{C_{2}} \frac{\nabla \psi_{1}}{h_{1} h_{2}} \cdot \vec{n} d s}{\underset{C_{q 2}}{\mathscr{C}_{a}} \frac{\nabla q_{2} \cdot \vec{n}}{h_{2}^{2}} d s} .
$$

Once the upper layer equilibrium streamfunction is known, this expression can be used, in principle, to compute the lower layer transport in the skirt region. Note that for an order one streamfunction in the upper layer we can anticipate from (3.4) an order one flow in the lower layer on the closed contours. Instead, on the open $q_{2}$ contours the lower layer streamfunction will be $O\left(\lambda_{i}\right)$.

\section{a. The meridional island}

We first consider the case of a thin island oriented in the north-south direction as in Figure 1, surrounded by a topographic skirt confined to the lower layer. The skirt is assumed to be tall enough to produce closed pv contours girdling the island. Since the outermost closed pv contour, starting a $x=x_{T}$ and $y=y_{n}$ satisfies the relation

$$
\begin{aligned}
& \frac{f_{n}}{H_{2}}=\frac{f}{H_{2}-h_{T}\left(1-x / x_{T}\right)} \\
& \Rightarrow \frac{x}{x_{T}}=1-\frac{H_{2}}{h_{T}}\left(1-\frac{f}{f_{n}}\right)
\end{aligned}
$$


the condition that it reaches the southern boundary without striking the island at $x=0$, is simply

$$
\frac{h_{T}}{H_{2}}>\frac{\beta\left(y_{n}-y_{s}\right)}{f_{n}} .
$$

For an island of length $1400 \mathrm{~km}$ and for $\beta=210^{-11} \mathrm{~m}^{-1}$ and $f_{0}=10^{-4} \mathrm{~s}^{-1}$, this yields a critical value of $h_{T} / H_{2}$ of about 0.25 . Topography lower than this will have all the potential vorticity contours blocked and the $O(1)$ motion, with respect to the friction parameters satisfying (3.1) will be expunged.

To simplify the analysis, we further assume that the skirt width $x_{T}$ is smaller than the island half-length, so that the contributions of the tip regions to the denominator of (3.4) can be neglected in a first approximation when compared to those coming from the two nearly meridional sides of the integration contour. We then find (see Appendix A), that, to leading order in $\beta x_{T} / f$,

$$
\oint_{C_{q 2}} \frac{\nabla q_{2} \cdot \vec{n}}{h_{2}^{2}} d s=-q_{2}^{4} \frac{s}{\beta}\left(\frac{f_{n}^{2}-f_{s}^{2}}{f_{n}^{2} f_{s}^{2}}\right)
$$

(here $s=h_{T} / x_{T}$ is the slope of the topography, and $f_{n, s}$ are the values of $f$ at the northern and southern tips of the island, where $y=y_{n, s}$ ).

For small dissipation, and outside of any western boundary layers, the upper layer streamfunction can be derived from the Sverdrup balance as long as the flow in the lower layer is order one, or less, with respect to the Sverdrup flow. For our wind stress given by (2.1), the Sverdrup streamfunction satisfies

$$
\beta \psi_{1 x}=\vec{k} \cdot \operatorname{curl} \vec{\tau}=2 \tau_{o} / r_{o},
$$

which must be solved in the four regions shown in Figure 2 (the island in the figure is the circular island but the regional domains are the same with obvious changes for the meridional island). In regions A, B, and C, (3.7) yields the Sverdrup solution

$$
\psi_{1}=\frac{2 \tau_{o}}{\beta r_{o}}\left[x-x_{e}(y)\right], x_{e}(y)=\left(r_{o}^{2}-y^{2}\right)^{1 / 2}
$$

whereas the solution in region $\mathrm{D}$, in the "beta shadow" west of the island, is

$$
\psi_{1}=\frac{2 \tau_{o}}{\beta r_{o}} x+\Psi_{1 I}
$$

where $\Psi_{1 I}$ is the island constant for the upper layer island, which is computed in Appendix B.

Using (3.8a,b), one can evaluate the integral in the numerator of (3.4), as the sum of three contributions: one from the portions of the pv contour on the two sides of the skirt, another from the jumps in the Sverdrup solution across the boundaries between region $\mathrm{D}$ and regions 
$\mathrm{A}$ and $\mathrm{C}$, and the final one from the portions of the contour lying in the semi-circular tip regions. An explicit computation, whose details are not given here for brevity, shows that the latter contribution is dominant in our geometry: the ratio of this contribution to the sum of the two others is of the order $f_{o} s x_{T} / h_{2} \beta r_{o}=O\left(f_{o} / \beta r_{o}\right)$, as long as the ratio $h_{T} / h_{2}$ is order one. Since in our ocean basin $f_{o} / \beta r_{o}$ is about an order of magnitude greater than one, it is therefore reasonable to neglect all forcing except the forcing at the two extreme ends of the island's skirt.

This result, which agrees fairly well with the numerical evidence described below, can be partly understood by looking more closely at the forcing, $\nabla \psi_{1} \cdot \vec{n}$. On the long sides of the topography we have

$$
\begin{aligned}
\nabla \psi_{1} & =\frac{2 \tau_{o}}{\beta r_{o}}\left[\hat{i}+\hat{j} \frac{y}{\left(r_{o}^{2}-y^{2}\right)^{1 / 2}}\right], \quad x>0 \\
\nabla \psi_{1} & =\frac{2 \tau_{o}}{\beta r_{o}} \hat{i}, \quad x<0 .
\end{aligned}
$$

Hence, the largest term of the forcing, $\left(\partial \psi_{1} / \partial x\right) n_{x}$, has the same size on the two sides, but different signs, and consequently cancels out in the integration, leaving only the term $\partial \psi_{1} / \partial y$ multiplied by the very tiny component of the normal in the meridional direction. Similarly, the jumps across the boundaries between region $\mathrm{D}$ and regions $\mathrm{A}$ and $\mathrm{C}$ represent narrow, zonally oriented boundary layers whose flow contributes to (3.4) only due to the inner product of the $\psi_{1}$ gradient in the $y$ direction with the small component of the normal in that direction and it too is very small. On the other hand, the forcing $\nabla \psi_{1} \cdot \vec{n}$ can be locally much larger in the tip regions, particularly to the northeast and southeast of the tips, where both the streamlines of $\psi_{1}$ and the pv contours in the lower layer tend to wrap around the island, and this yields a sizable contribution to (3.4). It is a remarkable feature of the lower layer flow in this geometry how a very localized region of strong forcing can produce a circulation on the closed potential vorticity contours that essentially shoots a substantial flow all around the entire island.

The tip contributions can be evaluated by introducing local radial coordinate systems centered at the island extremes $\left(0, y_{n, s}\right)$, and assuming that in these small semicircular domains the pv contours are nearly coincident with the bathymetry and hence are lines of constant radius $r$. Then, using the linear dependence of $h_{2}$ on $r$, we can write $r$ in terms of $q_{2}$. For the northern tip, we find

$$
r=x_{T}+\left(\frac{f_{n}}{q_{2}}-H_{2}\right) \frac{x_{T}}{h_{T}}
$$

and the corresponding forcing is

$$
\int_{0}^{\pi} \frac{\nabla \psi_{1} \cdot \vec{n}}{H_{1} h_{2}} r d \theta=\frac{2 \tau_{o}}{\beta r_{o} H_{1}} \frac{q_{2}}{f_{n}} \int_{0}^{\pi} r d \theta \frac{y \sin \theta}{\left[r_{o}^{2}-y^{2}\right]^{1 / 2}} .
$$


Since in this region $y=y_{n}+r \sin \theta$ and since $r$ is less than or equal to $x_{\mathrm{T}}$ we can replace $y$ everywhere in (3.11) by $y_{n}$ so that the contribution of the northern tip is approximately given by

$$
\int_{0}^{\pi} \frac{\nabla \psi_{1} \cdot \vec{n}}{H_{1} h_{2}} r d \theta=\frac{2 \tau_{o}}{\beta r_{o} H_{1}} \frac{q_{2}}{f_{n}} \frac{2 y_{n} x_{T}}{\left[r_{o}^{2}-y^{2}\right]^{1 / 2}}\left\{1+\frac{f_{n}}{q_{2} h_{T}}-\frac{H_{2}}{h_{T}}\right\} .
$$

A similar analysis for the southern semi-circular tip yields a similar result. Adding the two of them together, and using (3.6), gives the dominant contribution to (3.4), namely,

$$
\begin{aligned}
\frac{d \Psi_{2}}{d q_{2}} & =\left(\frac{\lambda_{i}}{\lambda_{i}+\lambda}\right)\left\{\frac{-4 \tau_{o}}{s H_{1} r_{o}}\right\} \frac{y_{n} x_{T}}{\left(r_{o}^{2}-y_{n}^{2}\right)^{1 / 2}} \frac{f_{n} f_{s}^{2}}{\left(f_{n}^{2}-f_{s}^{2}\right)} . \\
& \cdot\left[\frac{1}{q_{2}^{3}}\left(1+\frac{f_{n}}{f_{s}}\right)\left(1-\frac{H_{2}}{h_{T}}\right)+\frac{1}{q_{2}^{4}} \frac{2 f_{n}}{h_{T}}\right]
\end{aligned}
$$

Integrating (3.13) and using the condition that the lower layer streamfunction vanishes on the outer closed contour where $q_{2}=f_{n} / H_{2}$ yields $\psi_{2}$ as a function of the pv,

$$
\psi_{2}=\frac{A}{s}\left[\frac{1}{2}\left(1+\frac{f_{n}}{f_{s}}\right)\left(1-\frac{H_{2}}{h_{T}}\right)\left[1-\frac{f_{n}^{2}}{q_{2}^{2} H_{2}^{2}}\right]+\frac{2}{3} \frac{H_{2}}{h_{T}}\left(1-\frac{f_{n}^{3}}{q_{2}^{3} H_{2}^{3}}\right)\right]
$$

where, recall, $s=h_{T} / x_{T}$, and

$$
A=\left(\frac{\lambda_{i}}{\lambda_{i}+\lambda}\right)\left\{\frac{-4 \tau_{o}}{H_{1} r_{o}}\right\} \frac{y_{n} x_{T}}{\left(r_{o}^{2}-y_{n}^{2}\right)^{1 / 2}} \frac{f_{s}^{2}}{\left(f_{n}^{2}-f_{s}^{2}\right)} \frac{H_{2}^{2}}{f_{n}} .
$$

Figure 3 shows the form of $\psi_{2}(x)$ over the skirt at the mid latitude, $y=0$, for $h_{T}=0.5 \mathrm{H}_{2}$ (solid line) and $h_{T}=0.999 H_{2}$. The calculation is shown for an anticyclonic wind stress curl, which yields an anticyclonic circulation in the lower layer. Note that the abscissa $x / x_{T}$ falls a bit short of unity, because of the slope of the $q_{2}$ contours in the $x . y$ plane due to the $\beta$ effect. This leaves a small sliver of the edge of the skirt outside the closed $q_{2}$ contours. The weak solution over the flat region can be continued on the open $\mathrm{pv}$ contours over this sliver but for concision we will not discuss it in detail. When the maximum height of the skirt almost reaches the interface between the layers the form of the stream function profile is different and is flatter in the neighborhood of the island with strong variations of $\psi_{2}$ limited to the outermost closed potential vorticity contours nearer the edge of the skirt.

The total transport $\psi_{s}$ in the skirt region predicted by linear theory is obtained by evaluating (3.14) at $x=0$ and $y=y_{n}$. Rearranging terms a bit, we get

$$
\begin{aligned}
\psi_{s}= & \frac{\lambda_{i}}{\lambda_{i}+\lambda}\left(\frac{-\tau_{0}}{2 \beta}\right) \frac{H_{2}}{H_{1}} \frac{\left(x_{T} / r_{0}\right)^{2}}{\sqrt{1-\left(y_{n} / r_{0}\right)^{2}}} \\
& \times \frac{f_{s}^{2}}{f_{0} f_{n}}\left\{\frac{3 f_{n}}{f_{s}}-1+2\left(1-\frac{f_{n}}{f_{s}}\right) \frac{H_{2}}{h_{T}}+\left(\frac{1}{3}-\frac{f_{n}}{f_{s}}\right) \frac{h_{T}}{H_{2}}\right\}
\end{aligned}
$$




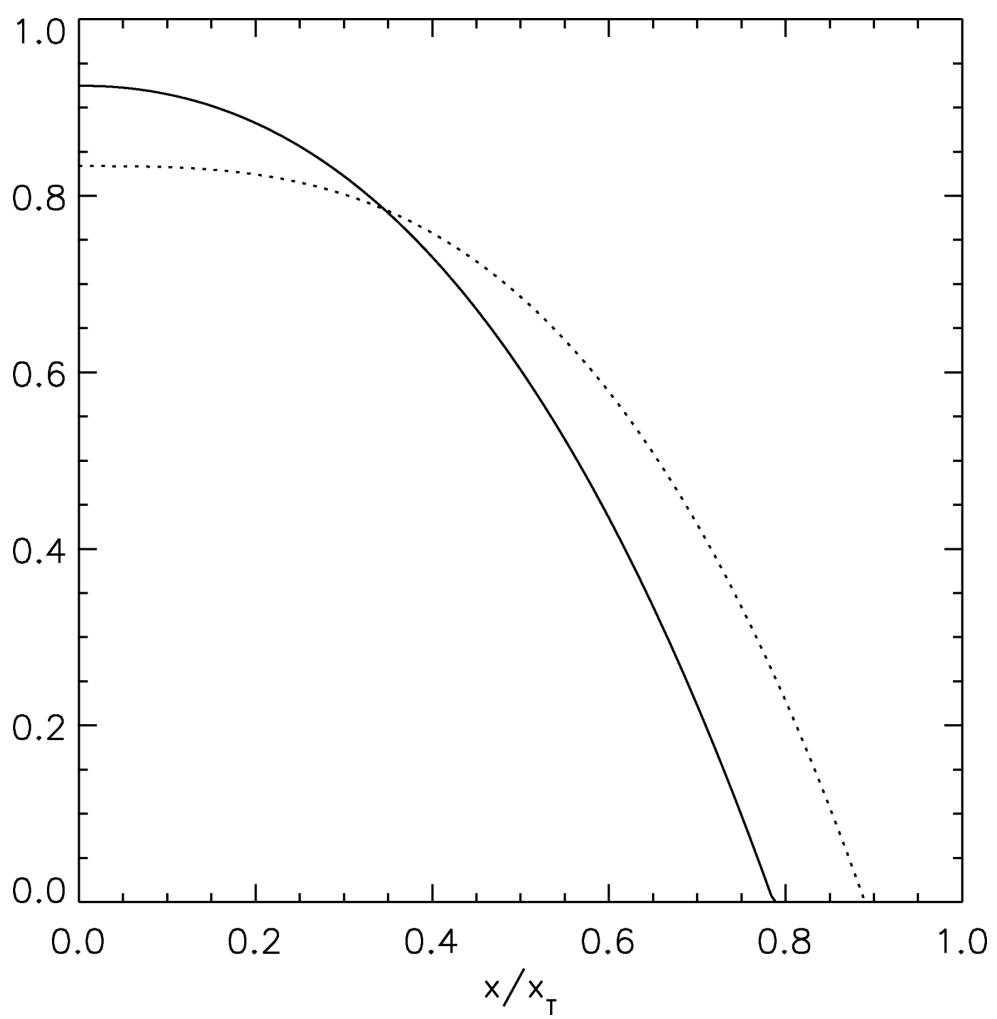

Figure 3. The form of the lower layer streamfunction as a function of $x$ at the mid latitude, $y=0$, for $h_{T}=0.5 H_{2}$ (solid) and $h_{T}=0.999 H_{2}$ (dotted).

which shows that the transport is proportional to the ratio $H_{2} / H_{1}$, and to the square of $x_{T} / r_{0}$, i.e., to the ratio between the total area of the tip regions and the basin area. The dependence on the basin radius is concentrated in the fourth fraction, and is manifestly a monotone decreasing one. That on the island length is more involved, but the numerical computation of Figure 4a shows that the transport decreases with increasing $y_{n}$ [note that increasing the island length increases the denominator of (3.4)].

The dependence of the lower layer transport on the skirt height is determined by the expression in the curly bracket in (3.15), which vanishes for some small value of $h_{T} / H_{2}$. This value is plotted in Figure $4 \mathrm{~b}$ (solid curve) as a function of $y_{n}$, for $\beta=210^{-11} \mathrm{~m}^{-1}$ and $f_{0}=10^{-4} \mathrm{~s}^{-1}$, together with the critical values (dotted line) obtained from (3.5c). The two curves almost coincide, providing an a posteriori check of the validity of the approximations made in deriving (3.15). Note also that in the limit $h_{T} / H_{2} \rightarrow 1$ the dependence on $f_{n} / f_{s}$ cancels out and the expression in the bracket tends toward a constant value of $2 / 3$. As shown in Figure 11, for short islands, one may get slightly higher values at intermediate values of $h_{T} / H_{2}$, yielding a transport that first increases, reaches a maximum, and then slowly 

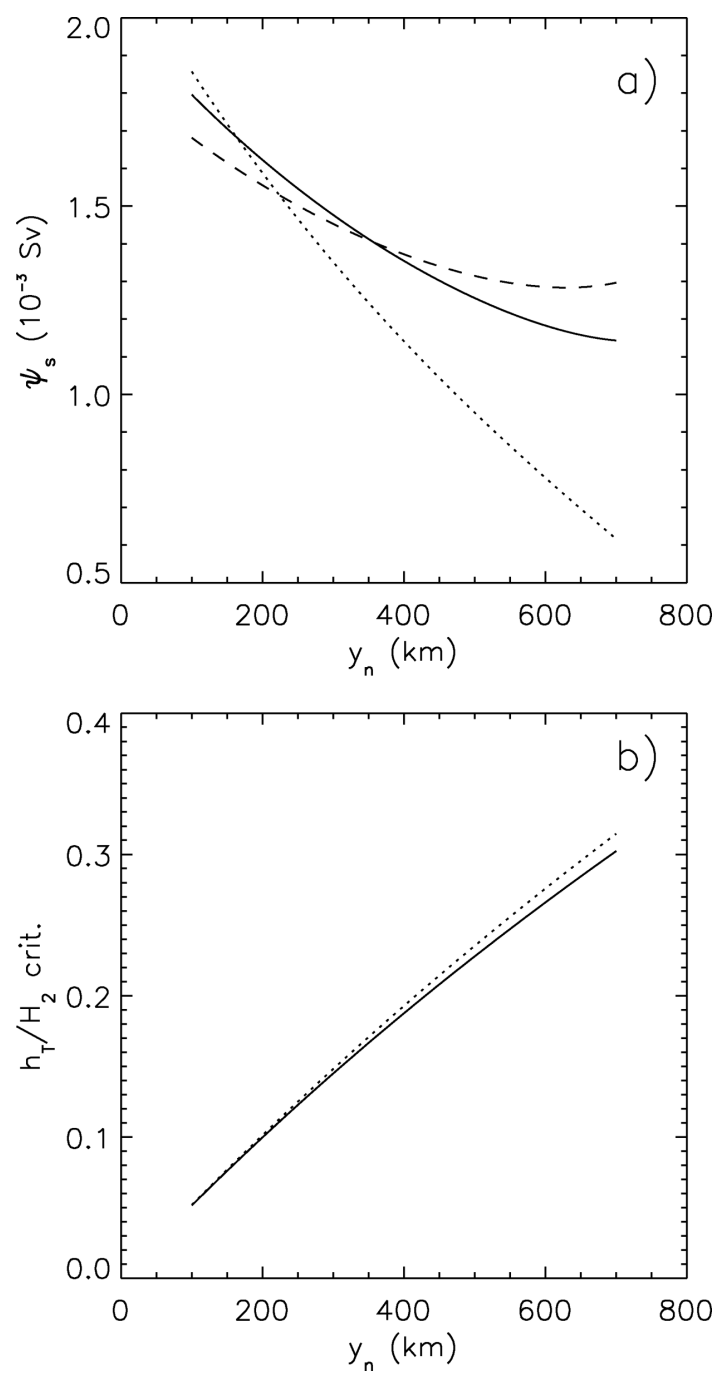

Figure 4. (a): the transport on the skirt in the lower layer as a function of the island half-length $y_{n}$, for $h_{T} / H_{2}=0.4,0.6$ and 0.8 (dotted, solid, dashed), as resulting from (3.15). The forcing and the other parameters are the same as in Fig. 9b): the dotted line gives the critical value of $h_{\mathrm{T}} / \mathrm{H}_{2}$ below which all pv contours on the skirt are blocked by the island, while the solid line gives the value of $h_{\mathrm{T}} / H_{2}$ for which the transport vanishes according to (3.15). Both values are shown as a function of $y_{n}$. The two curves are almost coincident.

decreases with $h_{T}$. However, the same figure shows that, in the range $0.5 \leq h_{T} / H_{2}<1$, the overall dependence of the transport on the skirt height is pretty weak.

If the topography is steep enough to intrude into the upper layer the situation becomes much more complex. However, in the region of the upper layer with steep topography 
and closed pv contours in the upper layer the flow on the closed contours is essentially barotropic and, as predicted in PINH, much stronger, $O\left(\lambda^{-1}\right)$. A full treatment of that problem is beyond the scope of the present paper. However, the important qualitative point is that the presence of stratification has shielded the lower layer from the direct effect of the wind forcing. The circulation on the closed $q_{2}$ contours is smaller than in the barotropic case although still much larger than in the flat region of the lower layer.

\section{b. The circular island}

In view of the weak projection of the forcing on the contour of integration we have turned to another island geometry, shown in Figure 2, consisting of a circular island of radius $r_{I}$, surrounded by a circular skirt of size $\Delta r=r_{T}-r_{I}$. The evaluation of (3.4) is more complicated for this configuration, and it is difficult to derive an accurate analytic expression for the lower layer transport. We believe, however, that the calculation below is sufficient to illustrate the main differences with the meridional island case.

In this geometry the pv contours in the skirt region are given by

$$
r\left(1-\frac{\beta \Delta r}{h_{T} q_{2}} \sin \theta\right)=r_{T}-\frac{\Delta r}{h_{T}}\left(H_{2}-\frac{f_{0}}{q_{2}}\right)
$$

where $(r, \theta)$ are polar coordinates with origin in the island center, and the radius $r$ varies in the range $r_{I} \leq r \leq r_{T}$. The threshold for closed pv contours is now obtained by asking that the outermost pv contour, on which $q_{2}=\left(f_{0}+\beta r_{T}\right) / H_{2}$, encircles the island, i.e, that on this contour $r$ is larger than $r_{I}$ at $\theta=3 \pi / 2$. This yields

$$
\frac{h_{T}}{H_{2}}>\frac{\beta\left(r_{I}+r_{T}\right)}{f_{0}+\beta r_{T}} .
$$

For $r_{I}=250 \mathrm{~km}, r_{T}=500 \mathrm{~km}, \beta=210^{-11} \mathrm{~m}^{-1}$ and $f_{0}=10^{-4} \mathrm{~s}^{-1}$, this corresponds to a critical value of $h_{T} / H_{2}$ of about 0.14 . It is also clear from (3.16) that the extent to which the pv contours deviate from circles depends on the size of the term $\beta \Delta r /\left(h_{T} q_{2}\right)$. For $h_{T} / H_{2}$ of order unity, the magnitude of this term is of the order of $10^{-2}$, and it seems reasonable to neglect it, to simplify the analysis. This amounts to neglecting the $\beta$ effect in the lower layer over the topography.

Let us now calculate the numerator in (3.4). As before, we must be aware of the regions with different Sverdrup solutions; in regions A, B, and C the solution is again that given in (3.8a), whereas in region $\mathrm{D}$ the solution is now given by

$$
\psi_{1}=\frac{2 \tau_{o}}{\beta r_{o}}\left(x-x_{w}(y)\right)+\Psi_{1 I}, \quad x_{w}=-\left(r_{I}^{2}-y^{2}\right)^{1 / 2}
$$

where $\Psi_{1 I}$ is the island constant, that is computed in Appendix B. 


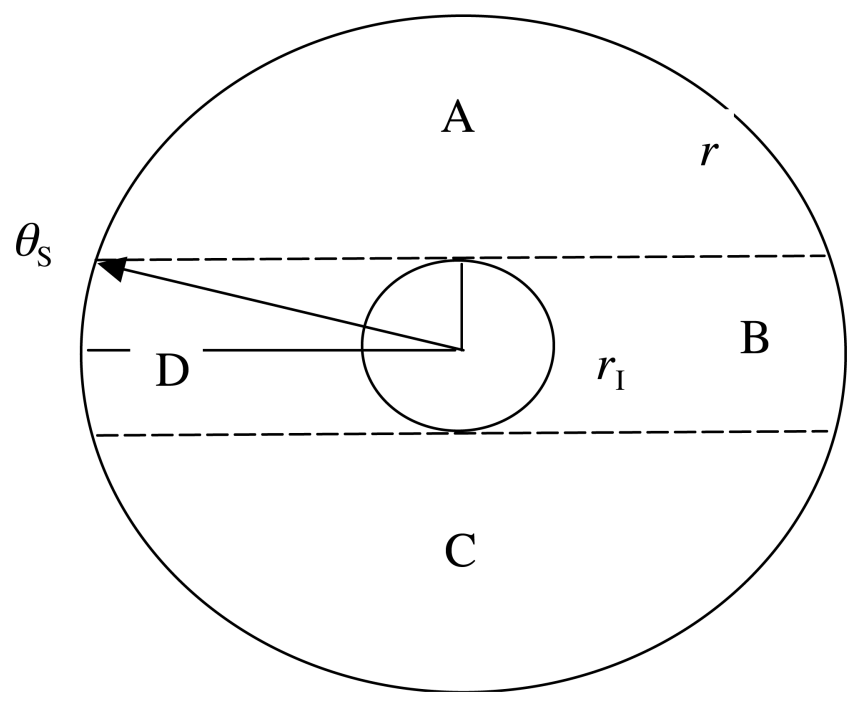

Figure 5. The outer circle is the contour of integration. The inner circle is the island in the upper layer. The angle $\theta_{\mathrm{S}}$ is the critical angle for the integration in Section $3 \mathrm{~b}$.

Since the pv contours are circles and the integrand in the numerator of (3.4) is north-south symmetric for weak forcing (see Pedlosky et al., 1997), we can carry out the integral on any circle of radius $r$ between the angles of 0 and $\pi$. In starting from the $x$ axis to the east of the island the integral proceeds counter clockwise until the contour intersects the boundary between regions $A$ and $D$. This occurs at an angle $\pi-\theta_{s}$ where, as seen in Figure 5,

$$
\theta_{S}=\sin ^{-1}\left(r_{I} / r\right)
$$

where the solution for $\psi_{1}$ in the integrand must be abruptly changed (note that as $r \rightarrow r_{I} \theta_{S}$ approaches $\pi / 2$ ). We will therefore do the integral in pieces, taking into account the delta function-like behavior of $\psi_{1}$. Since $\hat{r}=\hat{i} \cos \theta+\hat{j} \sin \theta$, where $\theta$ is the azimuth angle, we have in region $\mathrm{A}$

$$
\nabla \psi_{1} \cdot \vec{n}=\frac{2 \tau_{o}}{\beta r_{o}}\left[\cos \theta+\frac{y \sin \theta}{\left(r_{o}^{2}-y^{2}\right)^{1 / 2}}\right]
$$

while in region $\mathrm{D}$

$$
\nabla \psi_{1} \cdot \vec{n}=\frac{2 \tau_{o}}{\beta r_{o}}\left[\cos \theta-\frac{y \sin \theta}{\left(r_{I}^{2}-y^{2}\right)^{1 / 2}}\right]
$$

Note that the second term in (3.20b) may become very large and it is certainly greater than the equivalent term in (3.20a). In distinction to the meridional island the circular island's western coast is curved and introduces a new, relatively large term to the forcing. This is similar 
to the aforementioned case if the eastern boundary of the basin is convex facing westward rather than concave as for the circular basin. For the same curl the convex boundary of the western portion of the island acts the same way changing the sign of the zonal Sverdrup flow. The nature of the flow in the basin is a powerful function of the shape of the basin perimeter since in Sverdrup dynamics that information is propagated in a nondispersive manner westward.

In calculating the integrals needed in (3.4) we can use the fact that $y=r \sin \theta$ and the expression of $r$ as a function of $q_{2}$ obtained by setting $\beta$ to zero in (3.16). After a small calculation we obtain

$$
\begin{aligned}
& \frac{\oint_{C q_{2}} \frac{\nabla \psi_{1} \cdot \vec{n}}{h_{1} h_{2}} d s}{\frac{2 \tau_{o}}{f_{o} \beta r_{o} H_{1}}}=q_{2} r\left[2 \int_{0}^{\pi-\theta_{S}} \frac{\sin ^{2} \theta}{\left[m^{2}-\sin ^{2} \theta\right]} d \theta-2 \int_{\pi-\theta_{S}}^{\pi} \frac{\sin ^{2} \theta}{\left[n^{2}-\sin ^{2} \theta\right]} d \theta ;\right. \\
& n=r_{I} / r ; \quad m=r_{o} / r, \quad \theta_{S}=\sin ^{-1}\left(r_{I} / r\right)
\end{aligned}
$$

The two integrals in (3.21) yield terms of opposite signs. This is very much like the situation referred to earlier where there is a change in sign of forcing if the eastern boundary of the basin is convex rather than concave facing west. Here the term of opposite sign is introduced by the island geometry itself.

Including the contributions from the jumps in the solution across the boundary between regions $\mathrm{A}$ and $\mathrm{D}$ that are calculated in Appendix C, we finally obtain

$$
\begin{aligned}
& \frac{d \psi_{2}}{d q_{2}}= \\
& -\frac{\lambda_{i}}{\lambda+\lambda_{i}} \frac{2 \tau_{o}}{s \beta H_{1} r_{o}} \frac{f_{o}^{2}}{2 \pi q_{2}^{3}}\left[I_{m}-I_{n}-\frac{1}{r} \tan \left(\theta_{S}\right)\left(\left[r_{o}^{2}-r_{I}^{2}\right]^{1 / 2}-\frac{r_{o}^{2}}{r_{I}} \sin ^{-1}\left(r_{I} / r_{o}\right)-\frac{\pi r_{I}}{2}\right)\right]
\end{aligned}
$$

where $I_{m}$ and $I_{n}$ are the integrals appearing in the square bracket in (3.21), and $s=h_{T} / \Delta r$ is the slope of the skirt. Once $d \psi_{2} / d q_{2}$ is calculated the azimuthal transport around the pv contours in the lower layer is obtained by multiplying (3.22) by $d q_{2} / d r_{2}$, where

$$
\frac{d q_{2}}{d r_{2}}=-\frac{f_{0}}{h_{2}^{2}} s .
$$

Figure 6 shows the form of the azimuthal transport $\partial \psi_{2} / \partial r$ for an island for which $r_{I} / r_{T}=0.5$ and $r_{0} / r_{T}=5$ and for which the wind stress has a constant negative curl. The figure shows that the flow is everywhere anticyclonic. If we had ignored the contribution from the zonal boundary layers we would have obtained cyclonic circulation over part of the range in $r$ as a consequence of the forcing on the western curved boundary of the island (the integral $I_{\mathrm{n}}$ ). It is important to recall that a major part of the forcing, due to the equal meridional flow on both sides of the island cancels in the contour integral leaving the 


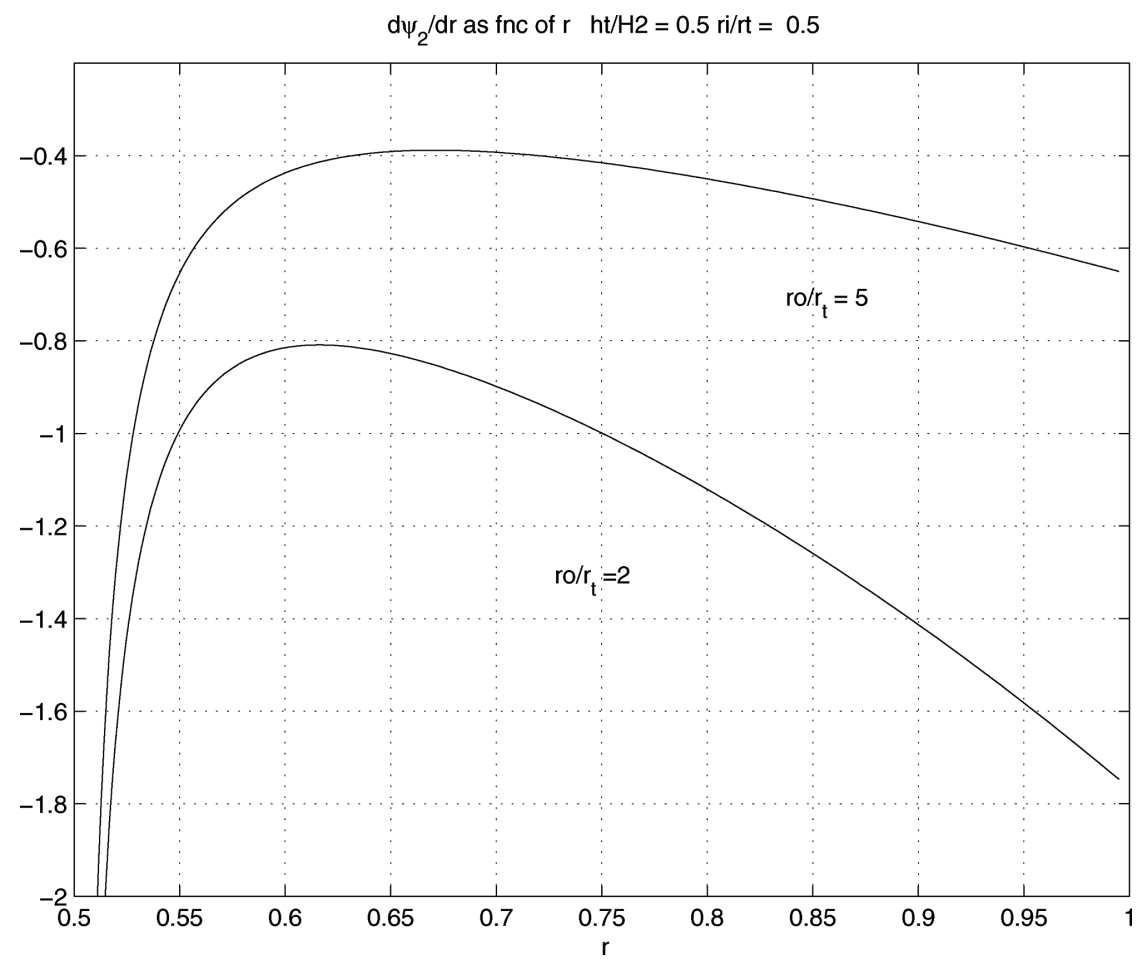

Figure 6. The azimuthal transport $\partial \psi_{2} / \partial r$ obtained from (3.22) and (3.23) for $r_{I} / r_{T}=0.5$ and $r_{o} / r_{T}=5$ and 2 .

residuals related to the curvature of the boundaries to determine the balance of forcing on the contours of potential vorticity. Figure 6 also shows a calculation for which the ratio of the basin radius to the skirt radius is smaller i.e. $r_{0} / r_{T}=2$. This enhances the effect of the integral $I_{m}$ and the flow is more strongly anticyclonic everywhere.

Figure 6 also illustrates one of the difficulties involved in this calculation. The azimuthal flow diverges when approaching the island border, because of the $\tan \left(\theta_{S}\right)$ appearing in the term that gives the contribution of the meridional jumps in the Sverdrup streamfunction to the forcing (remember that $\theta_{s}$ tends to $\pi / 2$ for $r \rightarrow r_{I}$ ). This shows that the simple arguments used in the appendix fail in the small region with complex dynamics near the top of the island (see Fig. 12 below). The failure, however, only affects the flow near the island, which can be expected to contribute very little to the total transport in practical cases. With this in mind, we may go on and use (3.22) and (3.23) to express the total transport $\psi_{s}$ as an integral over the radial extent of the skirt:

$$
\psi_{s}=\frac{\lambda_{i}}{\lambda_{i}+\lambda}\left(\frac{-\tau_{0}}{\pi \beta}\right) \frac{H_{2}}{H_{1}} \int_{\rho_{I}}^{\rho_{T}} d \rho\left[1-\frac{h_{T}}{H_{2}} \frac{r_{T}-r}{\Delta r}\right] F\left(\rho, \rho_{I}\right) .
$$




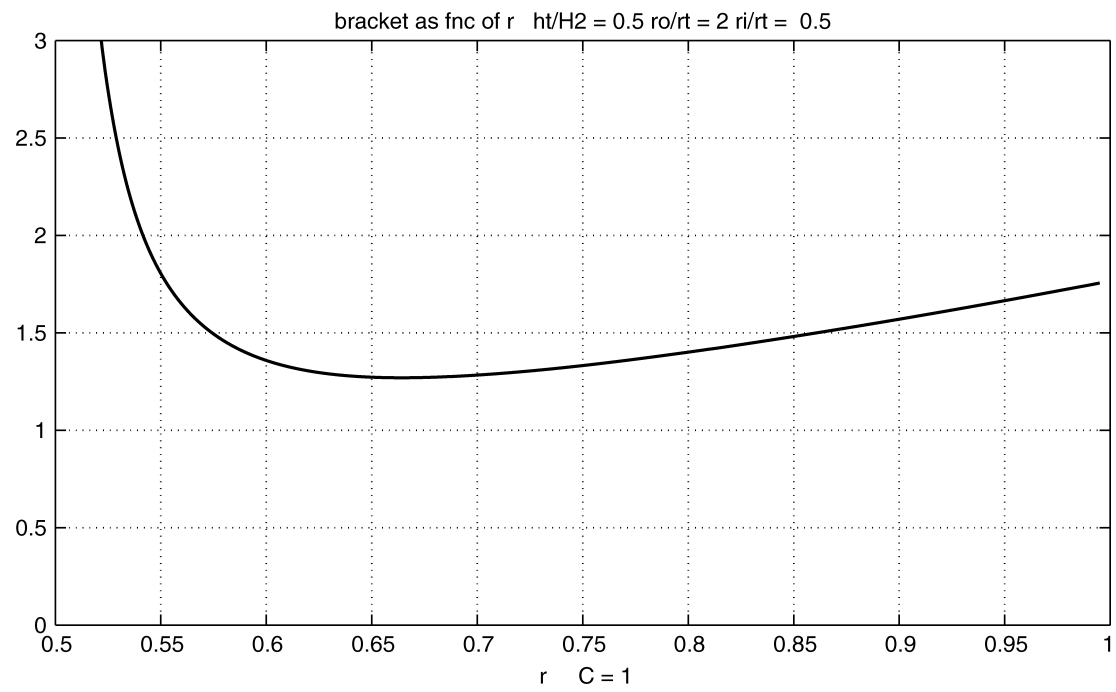

Figure 7. The square bracket in (3.22) for $r_{o} / r_{T}=2$.

Here $\rho=r / r_{0}, \rho_{I}=r_{I} / r_{0}, F$ is the term in square brackets in (3.22), and the terms in front of the integral are the same as in (3.15), except for a $\pi$ replacing a 2 in the denominator of the second fraction. Note that, just as in the meridional island case, the dependence on $s$ has canceled out, so that the transport does not depend explicitly on the skirt slope. There is a residual dependence on the skirt height, simpler than that occurring in (3.15): for a positive definite $F$, the transport predicted by (3.24) is linearly decreasing with $h_{T} / H_{2}$. This appears qualitatively consistent with the large $h_{T} / H_{2}$ behavior observed in the simulations (see Fig. $13 \mathrm{~b}$ below), but is certainly not right for small skirt heights, i.e., when we approach the threshold (3.17), since in this limit the transport should vanish. This is the price we have to pay for having neglected the beta-induced deformation of the pv contours, which was crucial in allowing a semi-analytic evaluation of (3.4).

For moderate skirt heights, however, we may expect (3.24) to be sufficient to roughly estimate the size of the transport. Since $F$ is slowly varying (see Fig. 7), except near the inner boundary, because of an unphysical divergence, we can approximately evaluate the integral in (3.24) by replacing $F$ with its radial average. This gives

$$
\psi_{s} \approx \frac{\lambda_{i}}{\lambda_{i}+\lambda}\left(\frac{-\tau_{0}}{\pi \beta}\right) \frac{H_{2}}{H_{1}}\langle F\rangle \frac{\Delta r}{r_{0}}\left(1-\frac{1}{2} \frac{h_{T}}{H_{2}}\right)
$$

with $\langle F\rangle$ of order unity, as shown by Figure 7. Note that, according to (3.25), the transport is proportional to $\Delta r / r_{0}$. This is different from the meridional island case, where the transport scaled with the square of $x_{T} / r_{0}$, and indicates that in the present geometry we may expect 
larger transports for the same skirt width. Likewise, we can estimate the ratio of the transport in the lower layer to the Sverdrup transport in the upper layer as

$$
\frac{\Psi_{s}}{\Psi_{I 1}}=O\left(\frac{\lambda_{i}}{\lambda_{i}+\lambda} \frac{H_{2}}{H_{1}} \frac{1}{2 \pi} \frac{\Delta r}{r_{0}}\left(1-\frac{1}{2} \frac{h_{T}}{H_{2}}\right)\right)
$$

that the flow can be expected to be somewhat smaller in the lower layer than in the upper layer although of the same order. For a barotropic model, as in PINH, the flow on closed $q_{2}$ contours would go as $\lambda^{-1}$, so as in the case of the meridional island, the principal effect of the stratification is to shield the lower layer from the direct forcing of the wind and reduce the response of the topographic resonance. Note also that the lower layer can equilibrate with no bottom drag. This differs from the potential vorticity homogenization approach of Dewar (1998), in which bottom drag is essential in determining the strength of the recirculation.

Given the delicacy of the relative balances of the residuals of the upper layer forcing on the lower layer, and the limitations of the analytic approach, it is important to compare these idealized linear results with numerical calculations which include the effects of lateral friction and nonlinearity.

\section{Numerical model and results for the linear regime}

The previous theoretical results indicate that deep circulations can be driven along topography that encircles an island through a fairly simple parameterization of coupling with the upper layer wind-driven flow. The circulation along a closed contour of potential vorticity results from a delicate balance between forcing from the upper layer and dissipation, in the form of bottom drag, interfacial stress, or horizontal viscosity. Owing to the symmetries in the problem, the net forcing on the deep layer is much weaker than the wind forcing in the upper layer, yet the mean circulation can be of similar strength. Numerous assumptions are required in order to make the problem analytically tractable and, while each are defensible, an independent confirmation of the result is desirable. In this section, the basic circulation patterns and parameter dependencies predicted by the theory are tested using a numerical model. The model contains more complete physics, and so provides an inherent validation of the analytic result, while the theory provides a clear interpretation of the balances that determine the mean circulation.

The numerical model used in this study is based on the Miami Isopycnal Coordinate Ocean Model (MICOM, Bleck et al., 1992). The model solves the primitive equations of motion using an isopycnal vertical coordinate. The MICOM solves prognostic equations for the isopycnal-layer averaged horizontal momentum, layer thickness (and sea surface height), temperature, and salinity. We have made several simplifications for the present problem. Temperature and salinity are uniform within each layer, and so the model effectively uses only the potential density field. There is no mixing of density (or tracers) between isopycnal layers and there is no surface buoyancy flux, so the model is adiabatic. All calculations reported here use two isopycnal layers. 
The initial layer thicknesses are $1000 \mathrm{~m}$ for both the upper and lower layers, giving a maximum total depth of $2000 \mathrm{~m}$. The horizontal grid spacing is $5 \mathrm{~km}$ for all cases. The Coriolis parameter varies linearly with latitude as $f=f_{0}+\beta y$, where $f_{0}=0.75 \times 10^{-4} \mathrm{~s}^{-1}$, $\beta=2 \times 10^{-11} \mathrm{~m}^{-1} \mathrm{~s}^{-1}$, and $y=0$ at the mid-latitude of the basin. The change in density between the two layers is $2.81 \mathrm{Kg} \mathrm{m}^{-3}$, resulting in a baroclinic deformation radius of $50 \mathrm{~km}$.

The upper layer is forced by a body-force representation of wind stress, as in (2.2a) with a uniform wind stress curl. The deep layer is forced through an interfacial stress term that is proportional to the difference between the upper layer velocity and the lower layer velocity, as in the linear theory (2.3b). Viscous dissipation is represented as a lateral Laplacian friction.

The relative strengths of the model forcing and dissipation are characterized by several boundary layer thicknesses. The strength of the wind stress is specified indirectly through the inertial boundary layer thickness, $\delta_{I}=\left(\frac{2 \tau_{o}}{r_{o} H_{1} \beta^{2}}\right)^{1 / 2}$. The strength of dissipation is specified through the previously defined frictional Munk boundary layer thickness.

For comparison with the linear theory, we choose relatively weak forcing so that the relative vorticity of the mean flow remains small and the large-scale mean circulation is stable to baroclinic and barotropic instabilities. Unless differently specified, the inertial boundary layer thickness is $\delta_{I}=2 \mathrm{~km}$, while the Munk layer thickness is $\delta_{M}=10 \mathrm{~km}$ $\left(\mathrm{A}=20 \mathrm{~m}^{2} \mathrm{~s}^{-1}\right)$, giving a ratio $\delta_{I} / \delta_{M}=0.2$. The interfacial drag coefficient in our standard runs is $\lambda_{i}=10^{-5} \mathrm{~m} \mathrm{~s}^{-1}$. Using the parameterization of baroclinic instability of Gent $e t a l$. (1995), this is equivalent to a horizontal thickness diffusion of $A_{h}=\lambda_{i} L_{d}^{2} / H=25 \mathrm{~m}^{2} \mathrm{~s}^{-1}$.

The model calculations are started from rest and run for a period of 20 years, at which point the fields are essentially steady.

\section{a. Meridional island}

Here we present numerical results for thin meridional islands in a circular basin, and compare them with the predictions of the linear theory previously developed. To summarize the expected dependences on the geometrical parameters, in Figure 8 we present a map obtained from (3.15) and (B.3), showing the lower layer transport on the skirt, normalized by the upper layer island constant, as a function of the basin radius $r_{o}$ and of the island half-length $y_{n}$, for a fixed value of the topography "aspect ratio," $x_{T} / y_{n}=0.5$. Qualitatively similar maps are obtained for higher (lower) values of the aspect ratio, with higher (lower) values of the transports, respectively. For fixed $r_{o}$, the transport increases monotonically with $y_{n}$ because of the dependence on $x_{T}^{2}$ in (3.15), while for given island length it decreases when going to larger basins, so that the maximum ratio of transports, just above $4 \%$, is obtained near the right lower corner of the map. These qualitative dependences are confirmed by the numerical simulations. In the following, we will restrict to a basin of $1000 \mathrm{~km}$ of radius.

A typical steady state obtained in the simulations is that of Figure 9, showing the streamfunctions of the two layers for a configuration with an island of $800 \mathrm{~km}$, surrounded by a $250-\mathrm{km}$ wide skirt that reaches $600 \mathrm{~m}$ of height. The forcing is from a constant curl wind 


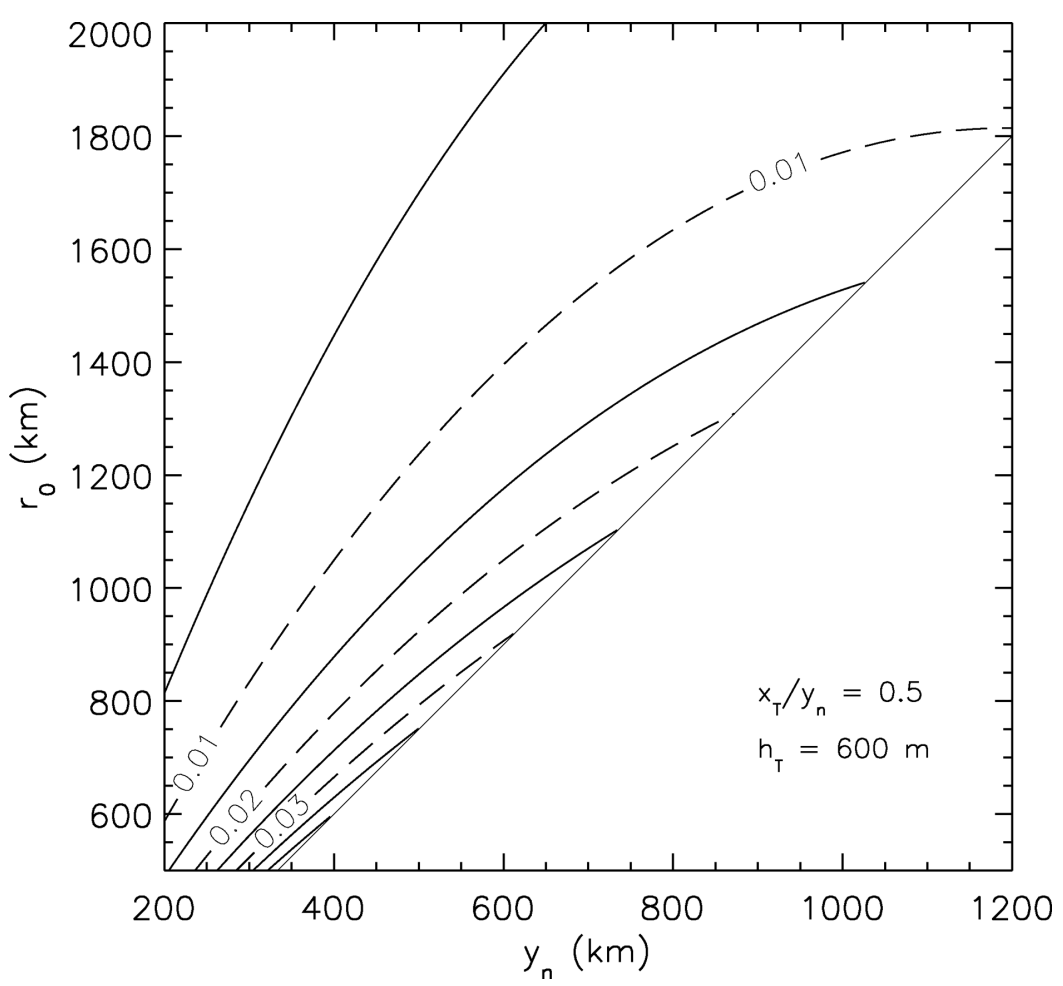

Figure 8. Lower layer transport over the skirt for the meridional island as predicted by (3.15). The transport is normalized by the upper layer island constant (B3), and is given as a function of the island half-length $y_{n}$ and of the basin radius $r_{0}$, for $h_{T}=600 \mathrm{~m}$ and $x_{T} / y_{n}=0.5$. The contour interval is 0.005 .

stress, whose size is determined by the non-dimensional parameters previously specified, that are appropriate for a linear context. The solution in the upper layer is very close to what may be expected on the basis of the barotropic linear theory developed in Pedlosky et al. (1997) with almost coinciding numerical and theoretical values of the island constant $\Psi_{I}$ $(0.0781 \mathrm{~Sv}$ and $0.0778 \mathrm{~Sv}$, respectively). This indicates that the interfacial drag coupling with the lower layer is small enough not to affect the upper layer dynamics (see the next subsection for a more detailed analysis of the effects of the interfacial drag on the circulation). On the other hand, the same coupling drives significant circulations in the lower layer, one of which is confined in the region of the western boundary layer, while the other, over the skirt, is characterized by streamlines that closely follow the PV contours encircling the island. Much weaker flow is observed over the rest of the flat bottom region, and the overall picture is in qualitative agreement with the theory. Moreover, the transport on the skirt of $1.277 \times 10^{-3} \mathrm{~Sv}$ is very close to the value of about $1.35 \times 10^{-3} \mathrm{~Sv}$ predicted by (3.15), indicating that the effects of lateral friction and of numerical dissipation are small. 

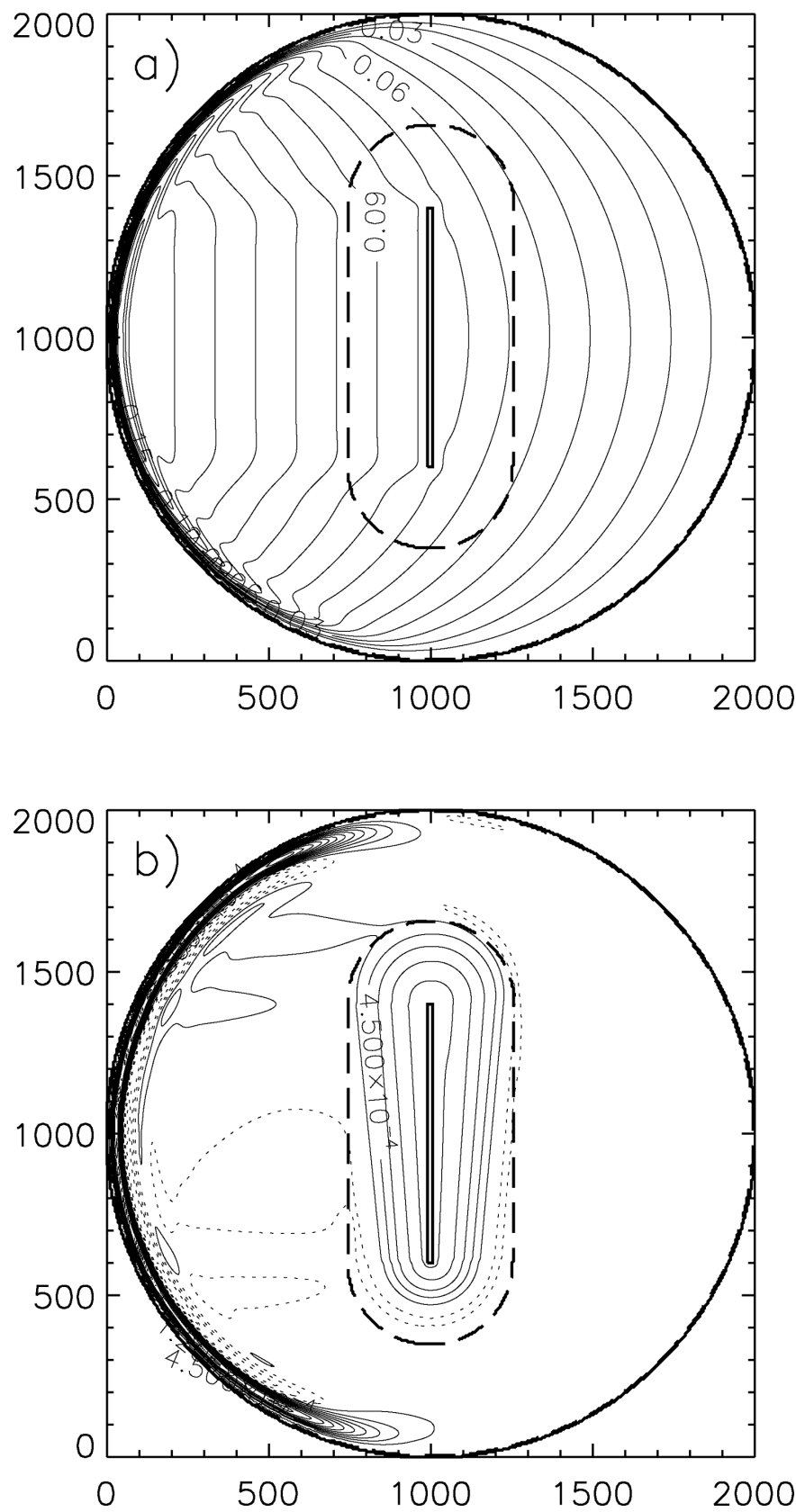

Figure 9. Steady state streamfunctions for the two layers (upper layer in panel a), from a simulation with an island of $800 \mathrm{~km}$, surrounded by a skirt of $250 \mathrm{~km}, 600 \mathrm{~m}$ tall, whose boundary is indicated by the dashed contour. The contour intervals are $0.01 \mathrm{~Sv}$ and $2.5 \times 10^{-4} \mathrm{~Sv}$, respectively. The lower layer pattern displays a closed circulation around the island, over the skirt, with streamlines closely following the contours of constant potential vorticity. 
An interesting point emerging from the analytic calculation of Section $3 \mathrm{a}$ is the observation that the main contributions to the transport appears to come from the regions of the skirt tips, which prompted us to retain only these contributions in the derivation of (3.15). As a consequence, the transport was found to be weakly decreasing with the island length (see Fig. 4a), mainly because of the increase in the denominator of (3.4). Two runs with the same geometry and parameters of Figure 9, but different island lengths $\left(y_{n}=300 \mathrm{~km}\right.$, and $y_{n}=600 \mathrm{~km}$ ), yield transports over the skirt of $1.6 \times 10^{-3} \mathrm{~Sv}$, and $0.68 \times 10^{-3} \mathrm{~Sv}$, respectively, confirm this behavior, supporting the idea that the forcing along the lateral portions of the PV contours is less effective than the one acting in the tip regions. This is further clarified in Figure 10, where we plot the integrand in the numerator of (3.4), $\nabla \psi_{1} \cdot \vec{n} / h_{1} h_{2}$, over the skirt region, as computed from the upper layer numerical solution of Figure 9, with some PV contours superimposed (dashed lines). The forcing is weak and cyclonic to the west of the island, and of the same order of the anticyclonic forcing present in the outer part of the eastern skirt. On the other hand, the anticyclonic forcing, that is prevalent in the tip regions, becomes very strong near the tips of island, and provides a major driving source for the circulation around the island, as suggested by the analytic estimates. It should be noted, however, that the picture emerging from the simulation is more complex, since near the eastern side of the island there is also an intense, but quite localized, forcing pattern due to the presence of a boundary layer, whose effects could not be included in the analytic approach. The effects of this boundary layer may be expected to become more important at larger values of the Munk layer thickness.

Another point to be explored is the dependence of the transport on the skirt height. Figure 11 shows that this dependence, for the geometry of Figure 9, is quite weak; the transport is somewhat lower for smaller and larger skirt heights, peaking around a height of $500 \mathrm{~m}$. The analytic dependence is also shown, for $y_{n}=300 \mathrm{~km}$ (dashed line), $400 \mathrm{~km}$ (solid line, that directly compares with the numerical values), and $600 \mathrm{~km}$ (dotted line). The agreement between the two is reasonably good for intermediate values of the skirt height, i.e., for $h_{T}$ in the range $400-700 \mathrm{~m}$, but theory tends to overestimate the transport outside this range. A possible explanation for this discrepancy is the neglect of the tip contributions in the denominator of (3.4). Taking into account these contributions should bring the solid line down a bit, and could even modify the functional dependence on the skirt height. A weak dependence of the transport on the skirt height was also found for other configurations, and we anticipate a similar behavior for the circular island case.

\section{b. Circular island}

The mean circulation for a circular island in a circular basin is shown in Figure 12. The island radius $r_{I}=250 \mathrm{~km}$, the topographic skirt radius is $r_{T}=500 \mathrm{~km}$, and the basin radius $r_{0}=1000 \mathrm{~km}$, giving $r_{i} / r_{T}=0.5$ and $r_{0} / r_{T}=2$. The skirt height is $500 \mathrm{~m}$, giving a topographic slope $s=0.002$. The streamfunction in each layer is nondimensionalized by the theoretical island constant (B.5). 


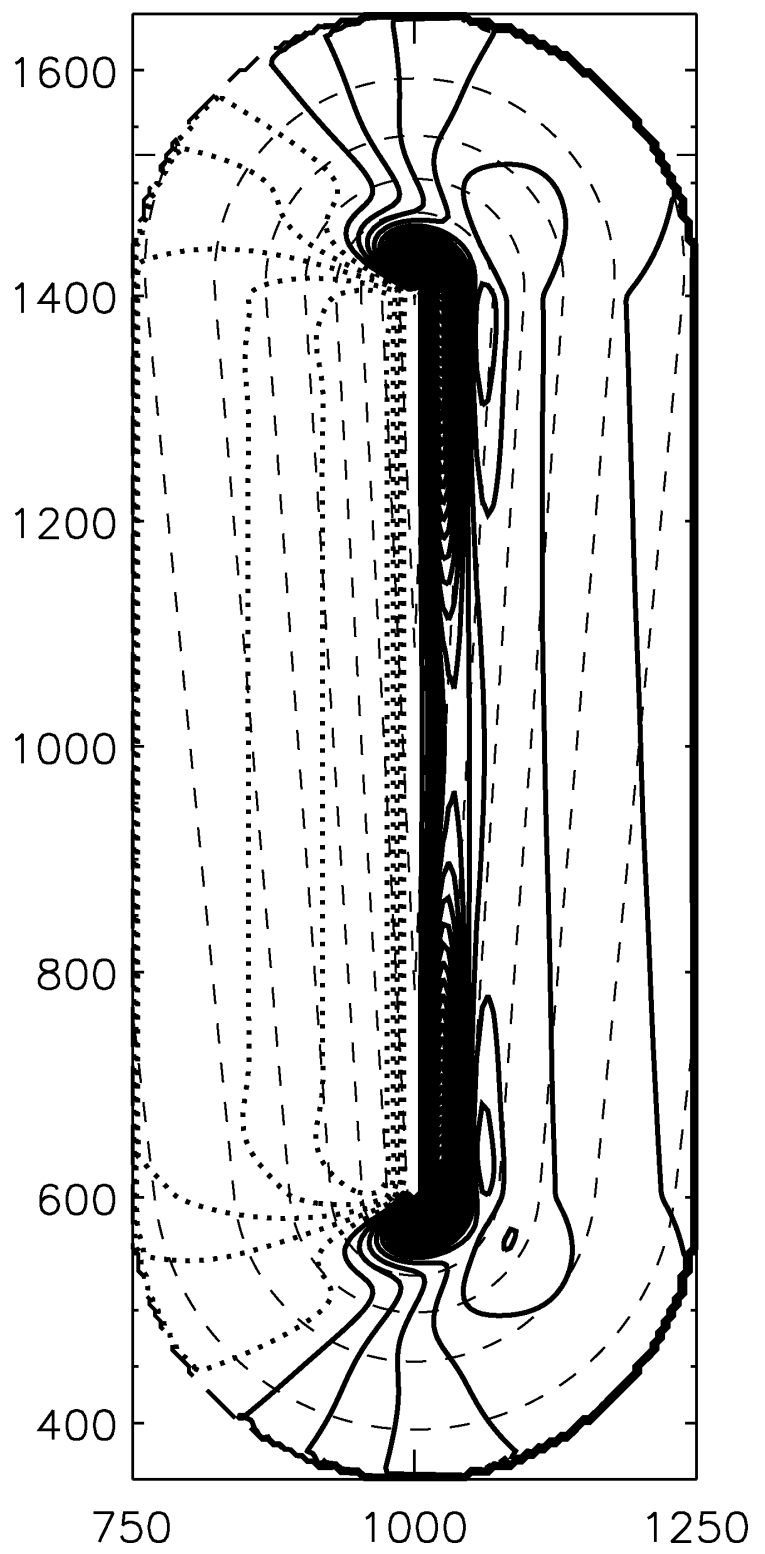

Figure 10. The integrand in the numerator of (3.4), measuring the strength of the forcing exerted by the upper layer, wind-driven dynamics, on the lower layer circulation. Equally spaced contours, with the solid (dotted) lines corresponding to anticyclonic (cyclonic) forcing, with the dashed lines indicating contours of constant potential vorticity. 


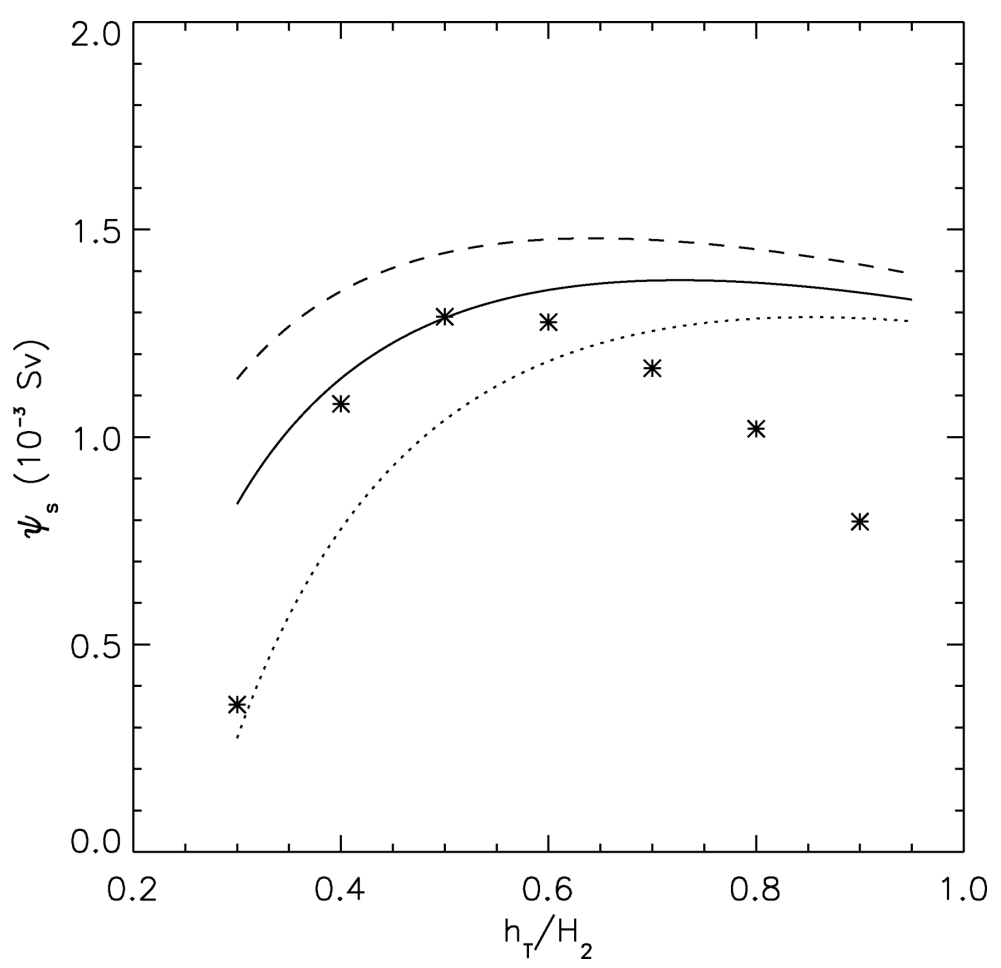

Figure 11. The dependence of the lower layer transport on the skirt height $h_{T}$ for the meridional island. Asterisks mark values from numerical simulations, with all other parameters as in Figure 9, while the curves are obtained from the analytic estimate (3.15). The solid line is for an island half-length $y_{n}=400 \mathrm{~km}$, as in the simulations, while the dashed (dotted) curve is for $y_{n}=300 \mathrm{~km}$ $\left(y_{n}=600 \mathrm{~km}\right)$.

The circulation in the upper layer reflects the Sverdrup transport away from the island, with the flow direction in regions $\mathrm{A}, \mathrm{B}$, and $\mathrm{C}$ tracing the shape of the eastern boundary. To the west of the island the zonal flow changes sign because the island west coast is convex, as expected from the linear theory. There is also a strong boundary layer along the eastern side of the island, which is required because the island constant is greater than the Sverdrup transport between the island and the eastern boundary, see (B.5). This eastern boundary layer was not included in the theory, but the westward boundary layers extending from the northern and southern tips of the island, also evident in the figure, were included. The influence of this eastern boundary layer is to drive an anticyclonic circulation within approximately $\delta_{M}$ of the island. This region close to the island is also where the theory predicts a very strong anticyclonic circulation driven by the northern and southern tip boundary layers. Because of the neglect of dissipation and this western boundary layer in the theory, we do not expect quantitative agreement between the model and theory close to the island. 

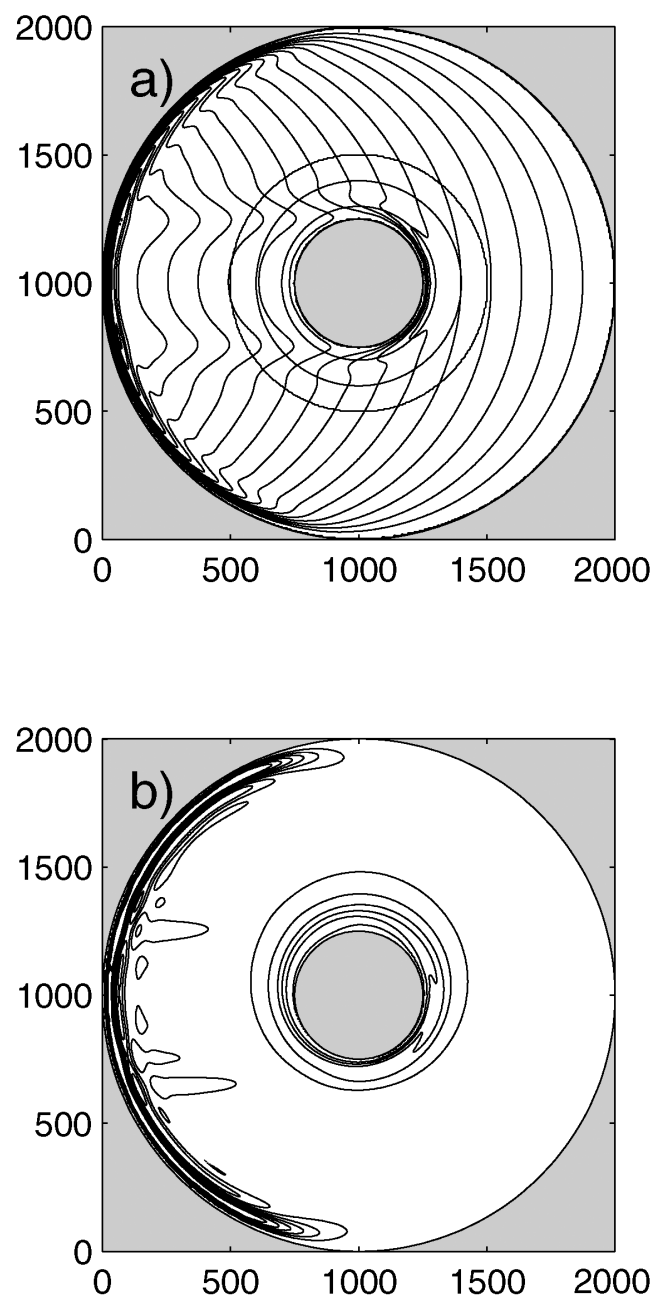

Figure 12. Mean streamfunction for (a) upper layer and (b) lower layer for $\delta_{M}=10 \mathrm{~km}, \delta_{I}=2 \mathrm{~km}$, $\lambda_{I}=10^{-5} \mathrm{~m} \mathrm{~s}^{-1}, r_{I}=250 \mathrm{~km}, r_{T}=500 \mathrm{~km}$, and $r_{o}=1000 \mathrm{~km}$. The streamfunction in both layers has been nondimensionalized by the upper layer island constant $\Psi_{I}=.095 \mathrm{~Sv}$, and the contour interval in a) is 0.1 and in (b) is .004 . The topography is indicated on (a) by the gray circular contours, contour interval $200 \mathrm{~m}$.

The model produces a mean anticyclonic circulation around the island of strength $2.18 \times 10^{-3} \mathrm{~Sv}$, or $0.023 \Psi_{I 1}$, which is somewhat lower than the value of $0.03 \Psi_{I 1}$ resulting from the rough estimate (3.26). However, the transport is 1.7 times that found in the standard experiment with the meridional island, in agreement with the analysis of Section $3 \mathrm{~b}$, indicating that for the same skirt width and basin geometry, transports should be larger for 
the circular island. Note that the transport is carried over a width of $O(250 \mathrm{~km})$, while the upper layer transport of $\psi_{1}$ is carried over $O(1000 \mathrm{~km})$ width, so the ratio of their transports per unit width is indeed of order unity.

The transport around the island is more than 2 orders of magnitude greater than the deep transport over the flat bottom, as expected from the theory, and confirms that even relatively weak coupling between the upper and deep layer can force an $O(1)$ flow over the closed topographic contours. There is a significant north-south asymmetry in the streamfunction because the potential vorticity contours do not coincide with topographic contours because of the influence of $\beta$. This contribution was neglected over the topographic skirt in the theory, and the numerical result demonstrates that its primary influence is to distort the circulation slightly but it does not change the qualitative response in the deep layer.

The primary purpose of the model is to verify the basic balances and parameter dependencies predicted by the theory. Expression (3.25) indicates that, for $\lambda=0$, the deep circulation over the skirt should be independent of $\lambda_{i}$. The circulation over the flat bottom is much weaker than the circulation over the skirt, but is expected to increase linearly with $\lambda_{i}$. A series of model calculations have been carried out in which the coupling coefficient has been varied, while all other parameters are the same as for the above standard case. The circulation strength over the skirt is shown as a function of the coupling coefficient in Figure 13a. The recirculation varies with the coupling strength approximately as $\left(\lambda_{i} / \beta h_{2}\left(r_{T}-r_{I}\right)\right)^{1 / 2}$. Recall that the theory assumes that this ratio is much less than 1 . The model requires lateral viscosity for computational stability and, as a result, we are not able to find a regime in which the solution is independent of the coupling strength. We can anticipate this general behavior from the theory (3.26), as dissipation (represented by $\lambda$ in the theory) will cause a decreasing recirculation strength with decreasing $\lambda_{i}$. The boundary layer width $\left(A h_{2} / \lambda_{i}\right)^{1 / 2}$ is $20 \mathrm{~km}$ for $\left(\lambda_{i} / \beta h_{2}\left(r_{T}-r_{I}\right)\right)=10^{-2}$, but increases to $O(300 \mathrm{~km})$ for the weakest coupling strength in Figure 13a. This viscous dissipation was not included in the theory, and for dissipation values required for computational stability in the numerical model $\left(A=20 \mathrm{~m}^{2} \mathrm{~s}^{-1}\right)$ as we move into the regime of small $\lambda_{i}$, the boundary layer becomes important.

The transport over the flat interior, between the topography and the eastern boundary, is always less than that over the topographic skirt, but it shows a stronger dependence (nearly linear) on the coupling coefficient. This result is consistent with the theory, and confirms that the dynamics over the region of closed potential vorticity contours are very different from that found in the region of blocked potential vorticity contours. Even for weak coupling in the presence of lateral dissipation the circulation over the closed topographic contours is much stronger than over the flat interior. The specific parameter dependence in Figure 13a is difficult to predict analytically, but the overall result, that the resonant circulation over the topography is intermediate to that for the barotropic flow and the flat interior, is robust to the details of the model physics.

Figure 13b shows the nondimensional transport as a function of $h_{T} / H_{2}$. The dependence is non-monotonic, and similar to that found in the meridional island case (see Fig. 11). For $h_{T} / H_{2}$ less than 0.2 the transport becomes very small. This is consistent with (3.17), which 

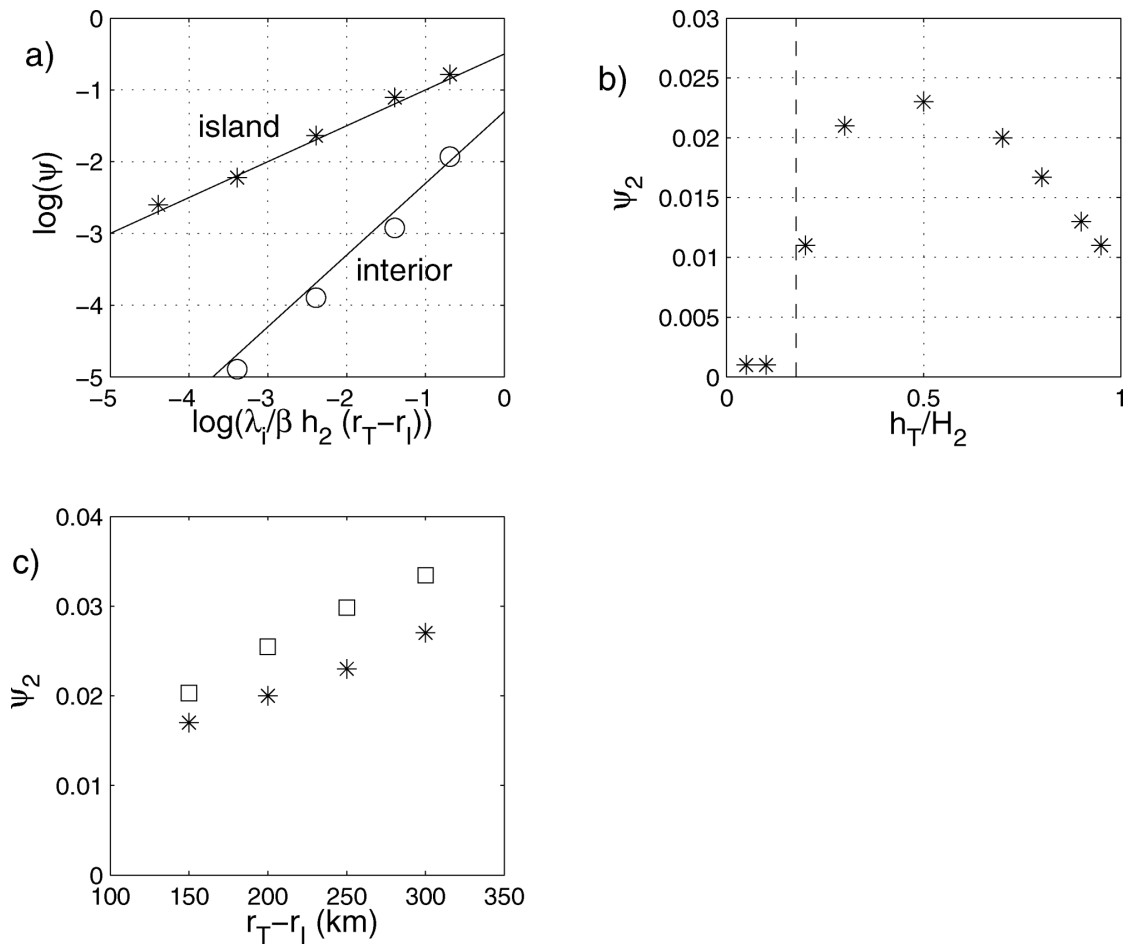

Figure 13. (a) The total nondimensional transport over the topographic skirt (asterisks) and flat interior east of the topography (circles) as a function of the scaled coupling coefficient $\lambda_{i} / \beta h_{2}\left(r_{T}-r_{I}\right)$. The straight lines have slopes proportional to $\lambda_{i}^{1 / 2}$ for the island result and $\lambda_{i}$ for the interior result. (b) The nondimensional transport over the skirt as a function of the bottom slope; the vertical dashed line is the approximate value for which the closed potential vorticity contours vanish. (c) The nondimentional transport over the skirt from different simulations in which the slope of the topography is held fixed, while the skirt height and size are varied. The numerical transports (asterisks) increase linearly with $r_{T}-r_{I}$, and are not far from those (squares) predicted by (3.26).

gives a threshold of 0.176 for this geometry, below which the region of closed potential vorticity contours is eliminated. For $h_{T} / H_{2}$ larger that 0.5 the transport decays, approximately linearly, consistently with the dependence predicted by (3.25), but the numerical slope is somewhat steeper than that resulting from (3.25).

Another prediction of theory is that the transport is not functionally dependent on the slope $h_{T} / \Delta r$. To test this prediction, we have performed runs with the same slope $\mathrm{s}=0.002$, but different couples $\left(h_{T}, \Delta r\right)$, whose transports are plotted in Figure 13c (asterisks), together with the corresponding transport values predicted by (3.26) (squares). The analytical and numerical transports are not far from each other, and they both increase almost linearly, consistently with the linear dependence on $\Delta r$ predicted by the theory. 
A last point worth exploring, at least partially, is that of how the delicate balances that determine the transport over the skirt may be affected by modifications of the upper layer circulation induced by changes in the basin geometry. As noted in Section $3 b$, in the meridional island case the circulation would be deeply affected by making the eastern boundary of the basin convex toward the island. In the circular island case, a similar effect may be induced by placing another island to the east of the one in consideration. This is illustrated in Figure 14, where the island has been moved a little toward the west (island A), and made smaller, to remain far enough from the circulation induced by the western boundary layer, while a larger island (B) has been placed in the eastern part of the basin. Panels a) and b) show the resulting equilibrium circulations, for the upper and lower layer, respectively. Note that circulations of opposite signs develop in the lower layer over the islands' skirts, due to the different structure of the upper layer flow to the east of the islands. To the east of $\mathrm{B}$, the flow is similar to that of the single island experiment of Figure 12, and the resulting forcing on the lower layer is likewise anticyclonic. On the other hand, to the east of A the curvature of the streamlines has changed from concave to convex, due to the presence of the convex western boundary of B. This destroys the anticyclonic contribution to the forcing from the eastern part of the island (the integral $I_{m}$ in (3.21)), allowing the net forcing on the lower layer flow to become cyclonic.

\section{Nonlinear numerical results}

The linear calculations in the preceding sections parameterized forcing of the deep ocean through a simple interfacial stress between the upper layer and the deep layer. This approach is intended to represent the tendency, in baroclinic fluids, for momentum to be transmitted from the upper ocean to the deep ocean as a result of baroclinic instability or vertical mixing. The advantage of such an idealized approach is that it allows for closed form analytic solutions and a straightforward interpretation of the driving mechanisms for the deep ocean.

It is, however, a drastic simplification of what is likely in the real ocean to be a much more complex process. Given the apparent sensitivity of the linear model to details of the forcing, it is important to confirm that the basic result remains relevant in more nonlinear regimes representative of the real ocean.

We have carried out a number of numerical model calculations in configurations similar to those already discussed, but with sufficiently strong forcing of the upper layer that the circulation becomes unstable and strongly time-dependent. The interfacial stress used for the linear calculations has been removed, and the only forcing on the deep layer comes from the resolved time-dependent motions. We report results from one of these calculations (although all have been found to produce qualitatively similar results) to demonstrate the basic response predicted by the simple linear models is also produced in a nonlinear model.

A calculation with a narrow meridional island, as in Section $4 \mathrm{a}$, has been carried out with $5 \mathrm{~km}$ horizontal resolution and forcing and dissipation characterized by an inertial boundary 

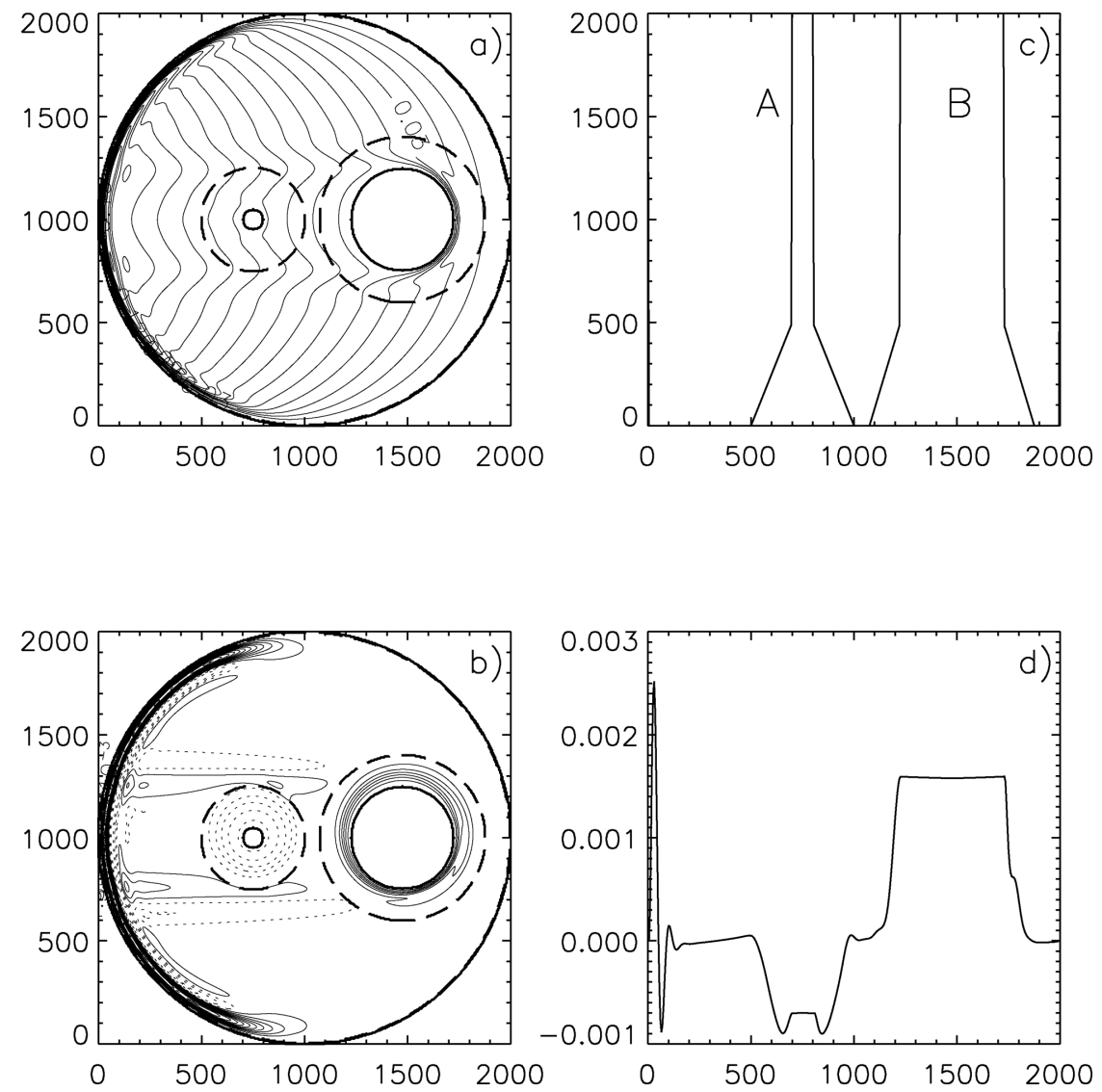

Figure 14. Equilibrium streamfunctions for the (a) upper layer and (b) lower layer, for a configuration with two islands, and anticyclonic forcing. Panels (c) and (d) show sections of the bathymetry and of the lower layer streamfunction at $y=0$. Note that circulations of opposite signs develop in the lower layer over the islands' skirts, due to the different structure of the upper layer flow to the east of the islands. To the east of $\mathrm{B}$, the flow is similar to that of the single island experiment of Figure 12 , and the resulting forcing on the lower layer is likewise anticyclonic. On the other hand, to the east of A the curvature of the streamlines is dramatically changed by the presence of the convex western boundary of $\mathrm{B}$, and the net forcing on the lower layer flow becomes cyclonic.

layer thickness of $15 \mathrm{~km}$ and a Munk boundary layer thickness of $10 \mathrm{~km}$. The stratification has been reduced so that the baroclinic deformation radius is $30 \mathrm{~km}$ and the large-scale flow is more susceptible to baroclinic instability. The model was started at rest and integrated for a period of 20 years.

The mean streamfunction over the final 5 years of integration in the upper and lower layers is shown in Figure 15. The upper layer circulation to the east of the island largely 

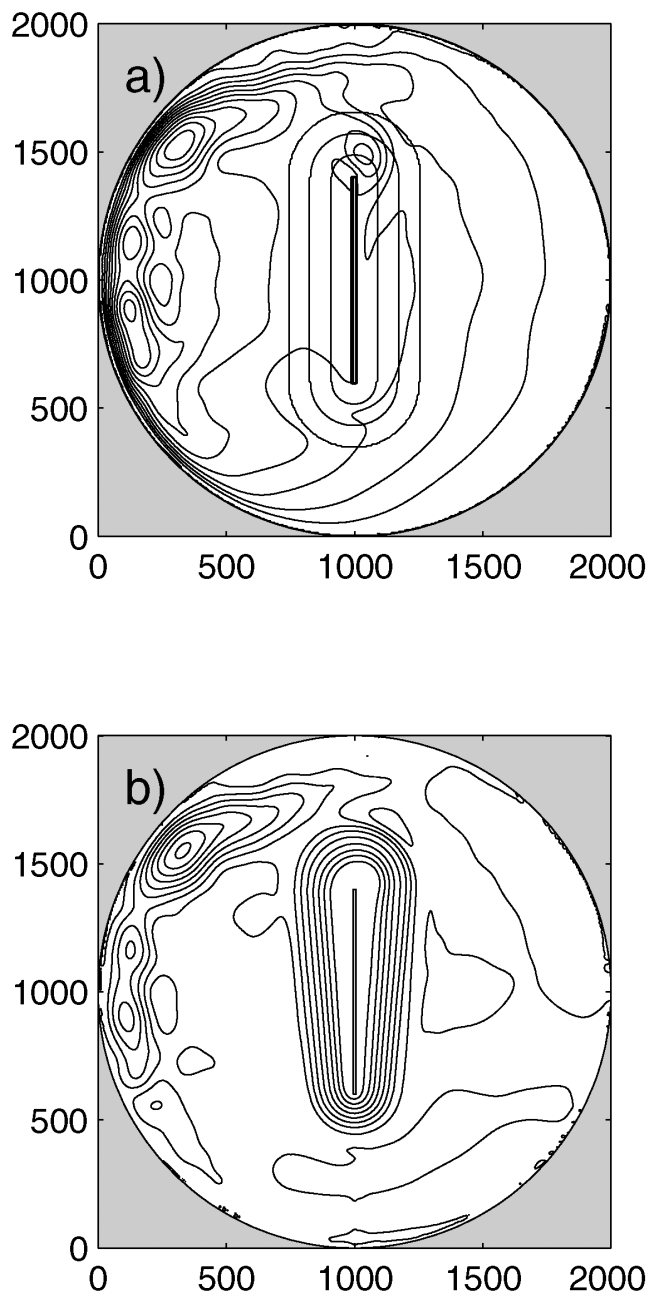

Figure 15. Mean (a) upper and (b) lower layer streamfunction from the final 5 years of a 20-year integration for the nonlinear numerical model. The streamfunction in both layers has been nondimensionalized by the upper layer island constant. The contour interval is 0.25 . The bottom topography is indicated in (a) by the gray contours, contour interval $200 \mathrm{~m}$.

reflects the expected Sverdrup flow, but there are considerable differences in the vicinity of and to the west of the island. The model produces strong, barotropic recirculation gyres on the offshore side of the western boundary current.

The flow in the western shadow of the island is similar to what one would expect from Sverdrup theory, however near the southern portion over the bottom topography, the flow turns to the north. There is a small closed anticyclonic recirculation near the northern tip of the island in the upper layer which we found in most nonlinear calculations. 
The deep layer is dominated by closed recirculations near the western boundary and around the skirt topography. The circulation around the island is 1.4 times the island constant in the upper layer, stronger than predicted by the linear theory but of the same order of magnitude as the upper layer wind-driven circulation. The flow around the island is strongly baroclinic, particularly along the western flank where the deep flow is in the opposite direction to the local wind-driven flow. Potential vorticity over the topographic skirt is not homogenized in layer 2 , but is instead dominated by the changes in layer thickness due to the topography.

The nonlinear model result is very complex and includes processes not considered in the linear theory (such as loss of momentum from the upper layer to the deep layer and eddydriven recirculation gyres). A detailed analysis of this regime is beyond the scope of the present study. It is encouraging, however, that the primary prediction from the linear theory, that the combined effect of closed $f / h_{2}$ contours in the deep layer and stratification produce deep recirculations of the same order as the upper layer wind-driven flow, is reproduced in this model that explicitly resolves the time-dependent forcing of the deep layer through instabilities of the upper layer flow.

\section{Discussion and conclusions}

The addition of both topography and baroclinicity to the dynamics of flow around planetary scale islands produces new and important qualitative changes in the circulation. The presence of baroclinicity, represented in our model by our two-layer system, introduces for the first time the issue of the vertical structure of the flow.

The presence of topography in the barotropic model in PINH introduced closed geostrophic contours around the island and the resulting flow, directly wind driven, could be very large since the geostrophic resonance on those contours led to velocities bounded only by the dissipation experienced by the flow on each closed contour. Most of that flow remained trapped to the topography and the Island Rule, which focused primarily on the interchange of flow between ocean basins on each side of the island, could be simply reinterpreted by applying it to the region defined by the outermost closed geostrophic contour instead of the island's surface boundary.

In the two-layer model, the flow in the lower layer is not directly forced by the wind but is instead forced by the action of the upper layer on the lower layer. In our linear models of Section 3, for example, the drag on the lower layer by the upper layer flow depends on the coupling constant $\lambda_{i}$ between the layers. When the coupling is weak, as seems realistic, the flow in the lower layer on the closed contours never exceeds the flow in the upper layer and, in linear theory, is reasonably independent of the value of the coupling as long as it is small. This is in contrast to the flat region outside the topographic skirt where the resulting lower layer flow is very weak and order $\lambda_{i}$ itself. Our numerical modeling of this linear regime shows a weak dependence on $\lambda_{i}$ over the topography, possibly as the result of lateral friction neglected in the theory and does show the linear dependence of the flat interior on $\lambda_{i}$. Thus the baroclinic model predicts a deep flow with a strength intermediate between the flow on 
the skirt in the barotropic model and the flow in the deep interior where there are no closed potential vorticity contours. In that sense the presence of closed potential vorticity contours provided by the topography allows a locally efficient mechanism to transfer momentum to the lower layer.

Furthermore, since the flow in the deeper layer is driven by the velocity of the upper layer Sverdrup flow rather than the wind stress itself, portions of that driving are ineffective in driving the flow. In particular, the meridional flow in our constant wind stress curl forcing is equal on each side of the island and cancels in the contour integral around the island that determines the net forcing that drives the deep response. This leads to a surprisingly sensitive dependence of the deep flow on the geometry of the island as well as the geometry of the basin. For example, in the case of the long, meridionally oriented island, the major driving comes from the upper layer flow acting on the relatively small northern and southern rounded tips of the island's topography. That localized forcing is responsible for the major impetus for motion over the entire length of the potential vorticity isolines in the lower layer. The forcing on the rest of the island's perimeter, although locally as strong, is almost completely self-canceling so that the total forcing is dominated by the effects of the tips. For the circular island the forcing is everywhere stronger but the convex shape of the western boundary of the island produces a local forcing that would actually drive a flow in the opposite direction to that imposed by the sense of the wind stress curl were it not for the contributions to the forcing of the zonally oriented boundary layers produced, in turn, by the discontinuities of the Sverdrup solution in the region west of the island. At the same time the forcing on the eastern side of the island depends on the curvature of the basin boundary and when that curvature is weak (on the meridional scale of the island), or non existent (in the case of a straight eastern boundary) there is no forcing provided by the eastern edge of the island. Thus the nature of the response to the wind forcing is a rather complex function of the basin and island geometry.

The strength of the circulation has been found not to be functionally dependent on the slope of the skirt: it depends from the skirt width and from the skirt height, but these functional dependences are different from each other. While the former is monotone increasing, the latter is nonmonotonic. If the skirt height is too small the presence of the planetary vorticity gradient allows the potential vorticity contours to strike the island's boundary instead of encircling it and the resulting deep circulation is very weak. For larger heights, the transport increases, reaches a maximum, and finally decreases, almost linearly, when the skirt height approaches the size of the lower layer.

The effects of lateral friction are neglected in the analytical theory of Section 3. Yet, the numerical experiments in the linear limit show no sign of baroclinic instability in the basin interior removed from the skirt and boundary currents. That implies that there is sufficient damping, due to lateral friction to expunge the growth rate of instabilities since the meridional flow should be unstable at all levels of shear if inviscid. That, in turn implies an intrinsic Reynolds number of order one on the deformation radius scale. The presence of this irreducible level of friction may contribute to the quantitative differences between inviscid theory and numerical experiment. 
If the island were large enough and if the curl varied substantially in the zonal direction across the breadth of the island an additional forcing would arise which we have not considered in this study.

Most of our numerical studies of the same models have been restricted to rather weak forcing in order to compare with the linear, analytic theory developed here. Preliminary results of a more nonlinear nature exhibit qualitatively similar enhanced circulation on the closed $f / h_{2}$ contours of the lower layer topography indicating a preferential pathway for the vertical transmission of momentum downward in that region.

Acknowledgments. This research was supported in part by a grant from the National Science Foundation (JP) NSF OCE 0925061 and (MAS) NSF OCE 0926656. We are delighted to be able to contribute to the volume honoring Professor Vladimir Kamenkovich for his lifetime of accomplishments in developing our understanding of the general circulation of the oceans.

\section{APPENDIX A}

Here we evaluate the denominator of (3.4) for the case of the meridional island. Over the topography on the long sides of the skirt

$$
\nabla q_{2}= \pm \frac{f s}{h_{2}^{2}} \vec{i}+\vec{j} \frac{\beta}{h_{2}},
$$

where the plus (minus) sign holds to the left (right) of the island, $\vec{i}$ and $\vec{j}$ are the unit vectors in the longitudinal and meridional direction, respectively, and $s=h_{T} / x_{T}$ is the slope of the topography. The outward normals on the right and left hand sides of the island are

$$
\vec{n}_{R}=\frac{\vec{i}-\vec{j} \beta h_{2} / f s}{\left(1+\left[\frac{\beta h_{2}}{f s}\right]^{2}\right)^{1 / 2}}, \quad \vec{n}_{L}=\frac{-\vec{i}-\vec{j} \beta h_{2} / f s}{\left(1+\left[\frac{\beta h_{2}}{f s}\right]^{2}\right)^{1 / 2}}
$$

and note that $f / h_{2}=q_{2}$ and so is constant along a pv contour.

On the right-hand side of the island

$$
\nabla q_{2} \cdot \vec{n}_{R}=-\frac{f s}{h_{2}^{2}}\left\{1+\left(\frac{\beta}{q_{2} s}\right)^{2}\right\}^{1 / 2}
$$

and using the fact that on that contour $d s=d y\left\{1+\left(\frac{\beta}{q_{2} s}\right)^{2}\right\}^{1 / 2}$ we find that the portion of the integral in the denominator of (3.4) on the right-hand sides of the island is:

$$
\int_{y_{s}}^{y_{n}} \frac{\nabla q_{2} \cdot \vec{n}_{R}}{h_{2}^{2}} d s=-q_{2}^{4} \frac{s}{\beta} \int_{y_{s}}^{y_{n}} \frac{\beta}{f^{3}} d y\left\{1+\left(\frac{\beta}{q_{2} s}\right)^{2}\right\}
$$

where we have ignored the relatively small $O\left(x_{T} / y_{n}\right)$ contributions from the island tips. 
The integral on the left hand side yields the same result so, ignoring only the northern and southern tips of the island the denominator in (3.4) yields

$$
\oint_{C_{q 2}} \frac{\nabla q_{2} \cdot \vec{n}}{h_{2}^{2}} d s=-q_{2}^{4} \frac{s}{\beta}\left(\frac{f_{n}^{2}-f_{s}^{2}}{f_{n}^{2} f_{s}^{2}}\right)\left\{1+\left(\frac{\beta}{q_{2} s}\right)^{2}\right\}
$$

Finally, we note that the last term in the curly bracket,

$$
\left(\frac{\beta}{q_{2} s}\right)^{2}=O\left(\frac{\beta h_{2} x_{T}}{f h_{T}}\right)^{2}=O\left(\frac{\beta x_{T}}{f}\right)^{2} \ll 1,
$$

can be neglected, giving the expression (3.6).

\section{APPENDIX B}

\section{The island constant}

It is shown in PINH that the island constant for single layer of fluid of constant depth, which will apply to the upper layer of our model in the linear limit is given by the following integrals

$$
\Psi_{1 I}=\frac{1}{\left(y_{n}-y_{s}\right)} \int_{y_{s}}^{y_{n}} \Psi_{\text {Sverdrup }}\left(x_{+}(y), y\right) d y-\oint_{C_{I}} \frac{\vec{\tau} \cdot \vec{t}}{\beta\left(y_{n}-y_{s}\right)} d s
$$

The first term on the right-hand side is the integral in $y$ of the Sverdrup streamfunction evaluated on the eastern boundary of the island.

\section{The meridional island of Section 3a}

The Sverdrup streamfunction for the thin, meridional island on its eastern side is, from (3.8a)

$$
\psi_{1}(0, y)=-\frac{2 \tau_{o}}{\beta r_{o}}\left(r_{o}^{2}-y^{2}\right)^{1 / 2}
$$

so that doing the integral in (B.1) leads us to

$$
\Psi_{1 I}=\frac{-2 \tau_{o}}{\beta r_{o}}\left[\frac{1}{2}\left(r_{o}^{2}-y_{n}^{2}\right)^{1 / 2}+\frac{r_{o}^{2}}{2 y_{n}} \sin ^{-1}\left(\frac{y_{n}}{r_{o}}\right)\right]
$$

for the case where $y_{s}=-y_{n}$ as in our model. 


\section{The circular island of Section $3 \mathrm{~b}$}

For the solution (3.18) the eastern boundary of the island boundary is $x_{+}=\left(r_{I}^{2}-y^{2}\right)^{1 / 2}$. Thus the first integral is

$$
\begin{aligned}
& \frac{1}{\left(y_{n}-y_{s}\right)} \int_{y_{s}}^{y_{n}} \frac{2 \tau_{o}}{\beta r_{o}}\left[\left(r_{I}^{2}-y^{2}\right)^{1 / 2}-\left(r_{o}^{2}-y^{2}\right)^{1 / 2}\right] d y, y_{n}=-y_{s}=r_{I} \\
& =\frac{2 \tau_{o}}{\beta r_{o}}\left[\frac{r_{I} \pi}{4}-\frac{1}{2}\left(r_{o}^{2}-r_{I}^{2}\right)^{1 / 2}-\frac{r_{o}^{2}}{2 r_{I}} \sin ^{-1}\left(r_{I} / r_{o}\right)\right]
\end{aligned}
$$

while the second term is easily calculated using Stokes theorem for the constant curl wind stress of our model and that term is just $-\frac{2 \tau_{o}}{\beta r_{o}}$, which when combined with (B.4) yields the island constant

$$
\Psi_{I 1}=\frac{2 \tau_{o}}{\beta r_{o}}\left[-\frac{1}{2}\left(r_{o}^{2}-r_{I}^{2}\right)^{1 / 2}-\frac{\pi}{4} r_{I}-\frac{1}{2} \frac{r_{o}^{2}}{r_{I}} \sin ^{-1}\left(\frac{r_{I}}{r_{o}}\right)\right]
$$

\section{APPENDIX C}

Here we compute the contribution to (3.4) from the jumps in the Sverdrup streamfunction across the boundaries between region D and regions A and C (see Fig. 2), for the circular island. Consider the jump across the line $y=r_{I}$. That jump in the $y$ direction is

$$
\psi_{1 A}-\psi_{1 D}=-\frac{2 \tau_{o}}{\beta r_{o}}\left\{\left(r_{o}^{2}-r_{I}^{2}\right)^{1 / 2}\right\}-\Psi_{1 I}
$$

That means that that in evaluating $\nabla \psi_{1} \cdot \vec{n}$ in the upper layer we will again approximate the gradient as a delta function, namely in the neighborhood of $y=r_{I}$

$$
\nabla \psi_{1}=-\vec{j}\left(\frac{2 \tau_{o}}{\beta r_{o}}\left\{\left(r_{o}^{2}-r_{I}^{2}\right)^{1 / 2}\right\}+\Psi_{1 I}\right) \delta\left(y-r_{I}\right)
$$

so that

$$
\nabla \psi_{1} \cdot \vec{n}=-\left[\frac{2 \tau_{o}}{\beta r_{o}}\left\{\left(r_{o}^{2}-r_{I}^{2}\right)^{1 / 2}\right\}+\Psi_{1 I}\right] \sin \theta_{S} \delta\left(y-r_{I}\right) ; \quad \theta=\pi-\theta_{S}
$$

Note that $\theta_{S}$ is positive. In (C.2b) $r$ is the circle on which the integral in (3.4) is being carried out. Now, our integral is an integral at fixed $r$ and so $d s$ is $r d \theta$. To evaluate the integral using the delta function we need to use the relation, for constant $r, r d \theta=d y / \cos \theta$. Keeping in mind that the integral in (3.4) is in the counterclockwise sense, it means that as an integral 
in $y$ the segment in the immediate neighborhood of $y=r_{I}$ is

$$
\begin{aligned}
\int \frac{\nabla \psi_{1} \cdot \vec{n}}{h_{1} h_{2}} d s & =\frac{-q_{2}}{H_{1} f_{o}} \int_{r_{I+}}^{r_{I-}}\left[\frac{2 \tau_{o}}{\beta r_{o}}\left[r_{o}^{2}-r_{I}^{2}\right]^{1 / 2}+\Psi_{1 I}\right] \frac{\sin \theta}{\cos \theta} \delta\left(y-r_{I}\right) d y \\
& =\frac{q_{2}}{H_{1} f_{o}} \tan \theta\left[\frac{2 \tau_{o}}{\beta r_{o}}\left[r_{o}^{2}-r_{I}^{2}\right]^{1 / 2}+\Psi_{1 I}\right]
\end{aligned}
$$

which, when combined with (B.5) yields

$$
\int \frac{\nabla \psi_{1} \cdot \vec{n}}{h_{1} h_{2}} d s=-\frac{q_{2}}{H_{1} f_{o}} \tan \left(\theta_{S}\right) \frac{2 \tau_{o}}{\beta r_{o}} 2\left(\frac{1}{2}\left[r_{o}^{2}-r_{I}^{2}\right]^{1 / 2}-\frac{1}{2} \frac{r_{o}^{2}}{r_{I}} \sin ^{-1}\left(r_{I} / r_{o}\right)-\frac{\pi r_{I}}{4}\right)
$$

as the contribution of both the northern and southern jumps in the streamfunction to the forcing of the lower layer flow. For small $r_{I} / r_{o}$ the first two terms in the final bracket in (C.4) nearly cancel, the residual being of order $-\frac{2}{3} \frac{r_{I}^{2}}{r_{o}}$ which would be much smaller than the last term in the bracket so that the contribution is dominated by the portion of the island constant related to the circulation of the stress around the upper layer island. In fact, for very large $r_{o}$, the variation of the distance from the island to the eastern boundary, over the latitude range of the island is negligible and the eastern boundary act as if it were straight contributing little to the integrals (3.21) and (C.4).

\section{REFERENCES}

Bleck, R.C., D. Hu and L.T. Smith. 1992. Salinity-driven thermocline transients in a wind-driven and thermohaline-forced isopycnal coordinate model of the North Atlantic. J. Phys. Oceanogr., 22, 1486-1505.

Dewar, W. K. 1998. Topography and barotropic transport control by bottom friction. J. Mar. Res., 56, 295-328.

Gent, P. R., J. Willebrand, T. J. McDougall and J. C. McWilliams. 1995. Parameterizing eddy-induced tracer transports in ocean circulation models. J. Phys. Oceanogr., 25, 462-474.

Godfrey, J. S. 1989. A Sverdrup model of the depth-integrated flow from the world ocean allowing for island circulations. Geophys. Astrophys. Fluid Dyn., 45, 89-112.

Pedlosky, J. 2010. Baroclinic flow around planetary islands in a double gyre: A mechanism for crossgyre flow. J. Phys. Oceanogr., 40, 1075-1086.

Pedlosky, J., L. J. Pratt, M. A. Spall and K. R. Helfrich. 1997. Circulation around islands and ridges. J. Mar. Res., 55, 1199-1251.

Pedlosky, J., R. Iacono, E. Napolitano and K. R. Helfrich. 2009. The skirted island: The effect of topography on the flow around planetary scale islands. J. Mar. Res., 67, 435-478.

Spall, M. A. 2000. Buoyancy forced circulations around island and ridges. J. Mar. Res., 58, 957-982.

Received: 28 February, 2011; revised: 30 September, 2011. 